\title{
Bayesian Assessment of Lorenz and Stochastic Dominance
}

\author{
David Lander \\ Pennsylvania State University \\ David Gunawan \\ University of New South Wales \\ William Griffiths \\ University of Melbourne \\ Duangkamon Chotikapanich \\ Monash University
}

March 13, 2017

Address for correspondence:

William Griffiths

Department of Economics

University of Melbourne

Vic 3010, Australia

Email: wegrif@unimelb.edu.au 


\section{SUMMARY}

Because of their applicability for ordering distributions within general classes of utility and social welfare functions, sampling theory tests for stochastic and Lorenz dominance have attracted considerable attention in the literature. We contribute to this literature by proposing a Bayesian approach for assessing Lorenz and stochastic dominance. For two income distributions, say $X$ and $Y$, estimated via Markov chain Monte Carlo (MCMC), we compute posterior probabilities for (i) $X$ dominates $Y$, (ii) $Y$ dominates $X$, and (iii) neither $Y$ nor $X$ is dominant by counting the proportions of MCMC draws that satisfy the constraints implied by each of the alternatives. We apply the proposed approach to samples of Indonesian income distributions for 1999, 2002, 2005 and 2008. To ensure flexible modelling of the distributions, mixtures of gamma densities are fitted for each of the years. We introduce probability curves that depict the probability of dominance at each population proportion and which convey valuable information about dominance probabilities for restricted population proportions relevant when studying poverty orderings. The dominance probabilities are compared with $p$-values from some sampling theory tests; the probability curves are used to gain insights into seemingly contradictory outcomes.

Keywords: Dominance probabilities, poverty comparisons, MCMC, gamma mixture.

JEL classification: C11, C12, D31, I32 


\section{INTRODUCTION}

Statistical tests of dominance regularly appear in the economics and finance literature. They employ statistical methods to compare the distributions of two random variables (or one random variable at two points in time) in such a way as to determine if one 'dominates' another. These tests have been used to compare income distributions in welfare analysis, distributions of asset returns in portfolio analysis, and risk analyses in actuarial science. We focus on Lorenz, stochastic and poverty dominance in income distributions and, in contrast to the existing literature, we propose a Bayesian approach to assessing dominance. Applications of stochastic dominance to asset returns in portfolio analysis can be found in Wong et al. (2008), Sriboonchitta et al. (2010) and Bai et al. (2011); for applications in actuarial sciences and risk analysis, see Kaas et al. (1994) and Denuit et al. (2005). Maasoumi and Millimet (2005) use stochastic dominance tests to examine trends in environmental quality.

The theoretical foundations for using Lorenz and stochastic dominance to analyse social welfare were laid by Atkinson (1970) and Shorrocks (1983). Among other things, it was shown that, for the class of monotonically increasing concave, but otherwise arbitrary, income utility functions, generalized Lorenz dominance (which is equivalent to second-order stochastic dominance) implies, and is implied by, a social welfare ordering. Robustness of stochastic dominance criteria to changes in the functional form of the social welfare function allows practitioners to avoid specifying a functional form for the utility or poverty function, and provides a compelling reason for its use in analyses of social welfare. Other inequality measures, such as the Gini coefficient and Atkinson's inequality index are useful when income distributions cannot be ordered according to stochastic dominance criteria, but they involve placing more restrictive assumptions on the functional form of the social welfare function or poverty index. Details of these various concepts, and the relationships between 
them, can be found in Atkinson and Bourguignon (1982), Lambert (2001), and Maasoumi (1997).

Empirical studies of stochastic, Lorenz and poverty dominance have typically been undertaken in a sampling-theory framework using nonparametric hypothesis tests. Tests for stochastic dominance have been proposed, developed, extended and/or applied by Bishop et al. (1989), McFadden (1989), Kaur et al. (1994), Bishop et al (1995), Anderson (1996), Davidson and Duclos (2000, 2013), Maasoumi and Heshmati (2000, 2008), Barrett and Donald (2003), Linton et al. (2005), Horváth et al. (2006), Linton et al. (2010), Berrendero and Cárcamo (2011), Bennett (2013) and Donald and Hsu (2016). Many of these papers also examine Lorenz dominance and poverty orderings. Additional papers concerned with Lorenz dominance include Beach and Davidson (1983), Bishop et al. (1991), Dardanoni and Forcina (1999), Schluter and Trede (2002) and Barrett et al (2014). Tests specifically concerned with poverty dominance include those proposed by Tabri (2015) and Barrett et al. (2016). Extensions to multivariate scenarios include Duclos et al (2006), McCaig and Yatchew (2007), and Bennett and Mitra (2013).

In this paper we propose a novel Bayesian approach to assessing dominance, and illustrate how it can be applied to income distribution data. There are a number of differences between existing sampling theory approaches and our proposed Bayesian approach. Sampling theory approaches begin with specification of null and alternative hypotheses, with some tests specifying $X$ dominates $Y$ (say) as the null hypothesis, and others using $X$ does not dominate $Y$ as the null. To exhaust all possibilities, these hypotheses are often also reversed, specifying that $Y$ does or does not dominate $X$. A test statistic and its limiting distribution are derived and results are reported in terms of $p$-values. In our Bayesian approach we begin with flexible parametric specifications for both income distributions and compute posterior probabilities of dominance as the probability that the parameters of the income distributions lie in the 
constrained region implied by dominance inequalities. We compute the probability that $X$ dominates $Y$, the probability that $Y$ dominates $X$, and the probability that neither distribution is dominant. This general approach - computing posterior probabilities of parameters lying within constrained regions - has been used by Geweke (1988) to assess explosive and oscillatory behavior of real per capita GDP for 19 OECD countries, and by Geweke (1986) in a variety of other contexts. A possible criticism of our proposal for computing posterior probabilities of dominance is its dependencies on how the income distribution is modelled through the likelihood function, and the prior information that is placed on the unknown parameters. These dependencies can be minimized, however. Subjectivity from specification of prior information can be limited by using relatively uninformative priors and is unlikely to have a major impact given the relatively large sample sizes that are typically used to estimate income distributions. The choice of likelihood function is likely to be more critical. In preliminary work, two of us (Chotikapanich and Griffiths, 2006) found results that were sensitive to a choice between the Dagum and Singh-Maddala income distributions, and concluded that a relatively flexible likelihood function is necessary for robust results. To minimize the impact of the likelihood function, in this paper we choose a more flexible likelihood function: a mixture of gamma densities. The added flexibility achieved by a mixture makes the analysis more complex, however. We are confronted with the problem of choosing an arbitrary number of points to assess dominance across the $(0, \infty)$ interval, or having to employ numerical methods to invert the distribution function of the mixture so that the quantile function can be used and the analysis restricted to the $(0,1)$ interval. We propose numerical methods to resolve this issue, and introduce several refinements to earlier work. The techniques are illustrated using Indonesian household income distributions for the years 1999, 2002, 2005 and 2008. 
The remainder of the paper is structured as follows. Conditions for dominance and the proposed method for Bayesian assessment are described in Section 2. A gamma mixture model and its associated Markov chain Monte Carlo (MCMC) algorithm for drawing observations from the joint posterior density for the parameters of the mixture are presented in Section 3. In Section 4 we describe how the MCMC draws can be used to find corresponding values for the quantile function, the generalized Lorenz curve and the Lorenz curve for the mixture of gamma densities. Data and estimation results for some Indonesian income distributions are considered in Section 5. Dominance results and a comparison with some sampling theory results are presented in Section 6; some concluding remarks are offered in Section 7.

\section{DOMINANCE CONDITIONS AND BAYESIAN ASSESSMENT}

The aim of this section is twofold. In Section 2.1 we discuss the conditions for Lorenz and stochastic dominance. Bayesian assessment of the dominance relationships is discussed in Section 2.2.

\subsection{Dominance Conditions}

To introduce the dominance conditions, we consider an income distribution $X$ that is described by density and distribution functions $p_{X}(x)$ and $F_{X}(x)$, respectively, with finite mean income $\mu_{X}=E(X)$. A number of expressions have been used in the literature for the Lorenz curve that gives the proportion of total income earned by the poorest proportion $u$ of the population. The one that is useful for noting the equivalence of generalized Lorenz dominance with second-order stochastic dominance (see below) is

$$
L_{X}(u)=\frac{1}{\mu_{X}} \int_{0}^{u} F_{X}^{-1}(t) d t \quad 0 \leq u \leq 1
$$

For computing values of the Lorenz curve to use for Bayesian assessment of dominance, the most convenient expression is 


$$
L_{X}(u)=F_{X}^{(1)}\left[F_{X}^{-1}(u)\right]
$$

where $F_{X}^{(1)}(x)=\left(1 / \mu_{X}\right) \int_{0}^{x} t p_{X}(t) d t$ is the first moment distribution function for $X$. We say that an income distribution for $X$ Lorenz dominates (LD) a distribution for $Y$ (say), expressed as $X \geq_{L D} Y$, if and only if

$$
L_{X}(u) \geq L_{Y}(u) \quad \text { for all } 0 \leq u \leq 1
$$

While this definition is the typical one used in the economics literature (see, for example, Lambert (2001) and Barrett et al. (2014)), the definition used in much of the statistics literature follows the opposite convention, with $L_{Y}(u) \geq L_{X}(u)$ being the condition for $X \geq_{L D} Y$. See, for example, Kleiber and Kotz (2003). Since $L_{X}(u) \geq L_{Y}(u)$ implies higher welfare for distribution $X$ in the sense that, other thing equal, less inequality is preferred to more inequality, we refer to this condition as one where $X$ dominates $Y$. This is the case when the Lorenz curve of $X$ lies nowhere below that of $Y$ for all population proportions $u$. For two income distributions with the same mean income, Lorenz dominance implies greater utility with respect to all strictly increasing and concave social welfare functions.

Because Lorenz dominance considers only the degree of inequality and not the level of income, generalized Lorenz dominance (GLD) was introduced to recognize that higher levels of income are associated with higher levels of welfare. We say that $X$ generalizedLorenz dominates $Y$, written as $X \geq_{G L D} Y$ if and only if

$$
\mu_{X} L_{X}(u) \geq \mu_{Y} L_{Y}(u) \quad \text { for all } \quad 0 \leq u \leq 1
$$

For all strictly increasing and concave social welfare functions, GLD provides an unambiguous ranking of distributions where the generalized Lorenz curves do not intersect. Given the expression for the Lorenz curve in equation (1), the condition in (4) can also be expressed as 


$$
\int_{0}^{u} F_{X}^{-1}(t) d t \geq \int_{0}^{u} F_{Y}^{-1}(t) d t \quad \text { for all } 0 \leq u \leq 1
$$

Condition (5) can be viewed as the sum of the incomes of the bottom $u$ proportion in $X$ being at least as great as the corresponding sum for $Y$ for any population proportion $u$. Writing the relation for GLD in this way demonstrates its equivalence to second-order stochastic dominance (SSD). See, for example, Maasoumi (1997) or Kleiber and Kotz (2003, p.25). An equivalent condition for SSD is

$$
\int_{0}^{x} F_{X}(t) d t \leq \int_{0}^{x} F_{Y}(t) d t \quad \text { for all } 0<x<\infty
$$

A stronger condition for welfare improvement than SSD (or equivalently, GLD) is that of first-order stochastic dominance (FSD). The distribution for $X$ first-order stochastically dominates $Y$, written $X \geq_{F S D} Y$, if and only if

$$
F_{X}^{-1}(u) \geq F_{Y}^{-1}(u) \quad \text { for all } \quad 0 \leq u \leq 1
$$

In this case the level of income from distribution $X$ is greater than the level of income from distribution $Y$ for all population proportions $u$. Alternatively, $X \geq_{F S D} Y$, if and only if

$$
F_{X}(x) \leq F_{Y}(x) \quad \text { for all } 0<x<\infty
$$

First-order stochastic dominance implies greater utility for all social welfare functions that are strictly increasing. Concavity is no longer required. The higher the degree of dominance, the greater the number of restrictions that need to be imposed on the social welfare function and the weaker the dominance inequality. That is, first-order stochastic dominance implies second-order stochastic dominance, but not vice-versa. Therefore, if $X \geq_{F S D} Y$, the generalized Lorenz curve of $X$ will also lie everywhere above $Y$.

Foster and Shorrocks (1988) showed that unidimensional stochastic dominance conditions are equivalent to unidimensional poverty orderings. Let $z_{H}$ denote the "poverty line". First-order poverty dominance, in terms of the quantile function of the distribution, is 
defined as $F_{X}^{-1}(u) \geq F_{Y}^{-1}(u)$ for all $0 \leq u \leq F\left(z_{H}\right)$, with strict inequality for some values of $u$. This is equivalent to the statement that the level of income of individuals is always (weakly) greater in distribution $X$ than in $Y$ up to population proportion given by $F\left(z_{H}\right)$. Similarly, second-order poverty dominance is defined as $\int_{0}^{u} F_{X}^{-1}(t) d t \geq \int_{0}^{u} F_{Y}^{-1}(t) d t$ for all $0 \leq u \leq F\left(z_{H}\right)$, with strict inequality for some values of $u$. A poverty ordering can be thought of as a form of restricted dominance. Barrett et al. (2016) consider testing for poverty dominance in terms of poverty gap profiles - see, for example, Jenkins and Lambert (1997). For a given poverty line, this dominance criterion is equivalent to restricted generalized Lorenz dominance.

\subsection{Bayesian Assessment of Dominance Relationships}

We now examine how to assess the dominance conditions within a Bayesian framework. Given two parametric distributions, each with known parameter values, one way to assess each form of dominance (LD, GLD or FSD) is to compute $L(u), \mu L(u)$ and $F^{-1}(u)$ for both distributions for a grid of values for $u$ in the interval $(0,1)$. If the grid contains a relatively large number of values, and the dominance inequality being considered is satisfied for all those values, then it is reasonable to conclude that the condition is satisfied for all $u$, and hence dominance holds. Alternatively, for GLD and FSD one could compute $F(x)$ and $\int_{0}^{x} F(t) d t$ for both distributions for a grid of values of $x$ in the interval $\left(0, x_{\max }\right)$ where $x_{\max }$ is a value deemed to be sufficiently large, and examine whether the relevant inequalities are satisfied for all these values.

Suppose now that the distributions for $X$ and $Y$ have unknown parameter vectors $\theta_{X}$ and $\theta_{Y}$ that are estimated using income distribution data. Since these parameters are not known with certainty, any conclusion about whether one distribution dominates another 
cannot be made with certainty. In Bayesian inference, uncertainty about whether one distribution dominates another, or whether one function is greater than another at a particular point, can be expressed in terms of a probability statement. To obtain or estimate such probability statements, assume we have draws on $\theta_{X}$ and $\theta_{Y}$ from the two posterior densities $p\left(\theta_{X} \mid \boldsymbol{x}\right)$ and $p\left(\theta_{Y} \mid \boldsymbol{y}\right)$. Taking FSD as an example, it will be useful to distinguish between $\operatorname{Pr}\left[F_{X}^{-1}(u) \geq F_{Y}^{-1}(u)\right]$ for a given value of $u$ and $\operatorname{Pr}\left[F_{X}^{-1}(u) \geq F_{Y}^{-1}(u)\right]$ for all values of $0<u<1$. An estimate of the first probability is given by the proportion of draws of $\theta_{X}$ and $\theta_{Y}$ for which $F_{X}^{-1}(u) \geq F_{Y}^{-1}(u)$ for a given $u$. We will denote this probability by $\operatorname{Pr}\left[X \geq_{F S D} Y \mid u\right]$. The probability $X \geq_{F S D} Y$ for the range $(0,1)$ is given by the proportion of values $\theta_{X}$ and $\theta_{Y}$ for which the inequality holds for all values of $u$. We denote this probability by $\operatorname{Pr}\left[X \geq_{F S D} Y\right]$. In practice we can consider a grid of $u$ values within the interval $(0,1)$ and count the number of parameter draws where the inequality holds for all $u$ in the grid. Since

$$
\operatorname{Pr}\left[X \geq_{F S D} Y\right] \leq \min _{u} \operatorname{Pr}\left[X \geq_{F S D} Y \mid u\right]
$$

a finer grid can be taken in the region that counts: those values of $u$ where $\operatorname{Pr}\left[X \geq_{F S D} Y \mid u\right]$ reaches a minimum. Similar probability statements can be made for LD and GLD.

In our empirical application we used 999 values of $u$ between 0.001 and 0.999 with increments of 0.001 . Graphing $\operatorname{Pr}\left[X \geq_{F S D} Y \mid u\right]$ against $u$ - curves that we call "probability curves" - provides useful information about the range(s) of the income distribution that have the largest impact on the probability of dominance. The inequality in (9) means that a probability curve is a powerful tool for visually determining the probability of dominance over the whole range of population proportions as well as within sub-intervals of $u$. Examining a probability curve is particularly useful for assessing restricted intervals such as 
poverty dominance as well as the sensitivity of poverty dominance to specification of a poverty line.

Having found $\operatorname{Pr}\left[X \geq_{F S D} Y\right]$ we can reverse the process to find $\operatorname{Pr}\left[Y \geq_{F S D} X\right]$. The probability that neither $X$ nor $Y$ dominates is given by $1-\operatorname{Pr}\left[X \geq_{F S D} Y\right]-\operatorname{Pr}\left[Y \geq_{F S D} X\right]$.

Suppose that we have a sequence of $M$ draws on $\theta_{X}$ and a sequence of $M$ draws on $\theta_{Y}$, and we assess probability using $M$ pairwise comparisons. Estimates of the dominance probabilities may change with different orderings of the draws on $\theta_{X}$ and $\theta_{Y}$. To ensure that the probability of dominance is robust to the order of the $M$ parameter draws, we randomly rearrange the order of the draws and repeat the procedure $C$ times. This gives a set of $C$ dominance probabilities, $\operatorname{Pr}\left(X \geq_{F S D} Y\right)_{t}$ for $t=1,2, \ldots, C$, and a set of $C$ probability curves. We use the mean probability of dominance, $\overline{\operatorname{Pr}\left(X \geq_{F S D} Y\right)}=\sum_{t=1}^{C} \operatorname{Pr}\left(X \geq_{F S D} Y\right)_{t} / C$, as our estimate of the probability of dominance. We will also define the maximum and minimum values of the set of $C$ dominance probabilities as the upper and lower bounds, respectively, for the dominance probability estimate. These bounds give an indication of how sensitive the dominance probability estimate is to the choice of pairwise ordering procedure. We can perform similar operations on the set of $C$ probability curves to obtain bounds on the probability curve.

\section{BAYESIAN ESTIMATION OF A GAMMA MIXTURE}

Since the probability of dominance could depend heavily on the assumed functional forms for the income distributions, it is important to specify functional forms that are sufficiently flexible to fit well. Mixtures of densities generally have the ability to approximate any functional form. We chose a gamma mixture. The more popular lognormal was not used since its predictive density does not have moments if a noninformative prior is used. Our specification and estimation procedure follows that in Wiper et al. (2001). In this section we 
briefly summarize his model and the conditional posterior densities used for Markov chain Monte Carlo sampling.

An income distribution that follows a gamma mixture with $K$ components can be written as

$$
p(x \mid \mathbf{w}, \boldsymbol{\mu}, \mathbf{v})=\sum_{k=1}^{K} w_{k} G\left(x \mid v_{k}, v_{k} / \mu_{k}\right)
$$

where $x$ is a random income draw from the probability density function (pdf) $p(x \mid \mathbf{w}, \boldsymbol{\mu}, \mathbf{v})$, with parameter vectors, $\mathbf{w}=\left(w_{1}, w_{2}, \ldots, w_{K}\right)^{\prime}, \boldsymbol{\mu}=\left(\mu_{1}, \mu_{2}, \ldots, \mu_{K}\right)^{\prime}$, and $\mathbf{v}=\left(v_{1}, v_{2}, \ldots, v_{K}\right)^{\prime}$. The pdf $G\left(x \mid v_{k}, v_{k} / \mu_{k}\right)$ is a gamma density with mean $\mu_{k}>0$ and shape parameter $v_{k}>0$,

$$
G\left(x \mid v_{k}, v_{k} / \mu_{k}\right)=\frac{\left(v_{k} / \mu_{k}\right)^{v_{k}}}{\Gamma\left(v_{k}\right)} x^{v_{k}-1} \exp \left(-\frac{v_{k}}{\mu_{k}} x\right)
$$

Including the mean $\mu_{k}$ as one of the parameters in the pdf makes the parameterization in (11) different from the standard textbook one, but it is convenient for later analysis. Let $\mathbf{x}=\left(x_{1}, x_{2}, \ldots, x_{n}\right)$ be a random sample from (9), and let $Z_{1}, Z_{2}, \ldots, Z_{n}$ be indicator variables such that $Z_{i}=k$ when the $i$-th observation comes from the $k$-th component in the mixture. Then,

$$
P\left(Z_{i}=k \mid \mathbf{w}\right)=w_{k} \quad \text { for } k=1,2, \ldots, K
$$

with $w_{k}>0$ and $\sum_{k=1}^{K} w_{k}=1$. Conditional on $Z_{i}=k$, the distribution of $x_{i}$ is $G\left(v_{k}, v_{k} / \mu_{k}\right)$.

Following Wiper et al. (2001), the prior distributions for each of the parameters are

$$
\begin{gathered}
p(\mathbf{w})=D(\boldsymbol{\varphi}) \propto w_{1}^{\phi_{1}-1} w_{2}^{\phi_{2}-1} \ldots w_{K}^{\phi_{K}-1} \\
p\left(v_{k}\right) \propto \exp \left\{-\theta v_{k}\right\}, \quad k=1,2, \ldots, K \\
p\left(\mu_{k}\right)=G I\left(\alpha_{k}, \beta_{k}\right) \propto \mu_{k}^{-\left(\alpha_{k}+1\right)} \exp \left\{-\frac{\beta_{k}}{\mu_{k}}\right\}, \quad k=1,2, \ldots, K
\end{gathered}
$$


These prior densities combine nicely with the likelihood function and are sufficiently flexible to represent vague prior information which can be dominated by the sample data. Because we are interested in the gamma mixture distribution, not its individual components, identification of the components and ordering of the means or scale parameters are not required (Geweke, 2007). However, if identification of the components is desired, it can be imposed a priori using the restriction $\mu_{1}<\mu_{2}<\ldots<\mu_{K}$.

The conditional posterior densities for use in a Gibbs sampler are:

1.)

$$
P\left(Z_{i}=k \mid \mathbf{x}, \mathbf{w}, \mathbf{v}, \boldsymbol{\mu}\right)=\frac{p_{i k}}{p_{i 1}+p_{i 2}+\cdots+p_{i K}}
$$

where $p_{i k}=w_{k} \frac{\left(v_{k} / \mu_{k}\right)^{v_{k}}}{\Gamma\left(v_{k}\right)} x_{i}^{v_{k}-1} \exp \left\{-\frac{v_{k} x_{i}}{\mu_{k}}\right\}$;

2.)

$$
p(\mathbf{w} \mid \mathbf{x}, \mathbf{z}, \mathbf{v}, \boldsymbol{\mu})=D(\boldsymbol{\varphi}+\mathbf{n})
$$

where $\mathbf{z}=\left(k_{1}, k_{2}, \ldots, k_{n}\right)^{\prime}$ are the realized components for each of the observations, and $\mathbf{n}=\left(n_{1}, n_{2}, \ldots, n_{K}\right)^{\prime}$, with $n_{k}$ being the number of observations for which $Z_{i}=k$. Thus, $\sum_{k=1}^{K} n_{k}=n$;

$$
p\left(\mu_{k} \mid \mathbf{x}, \mathbf{z}, \mathbf{w}, \mathbf{v}\right)=G I\left(\alpha_{k}+n_{k} v_{k}, \beta_{k}+S_{k} v_{k}\right)
$$

where $S_{k}=\sum_{i: Z_{i}=k} X_{i}$

4.)

$$
p\left(v_{k} \mid \mathbf{x}, \mathbf{z}, \mathbf{w}, \boldsymbol{\mu}\right) \propto \frac{v_{k}^{n_{k} v_{k}}}{\left[\Gamma\left(v_{k}\right)\right]^{n_{k}}} \exp \left\{-v_{k}\left(\theta+\frac{S_{k}}{\mu_{k}}+n_{k} \log \mu_{k}-\log P_{k}\right)\right\}
$$

where $P_{k}=\prod_{i: Z_{i}=k} x_{i}$. This density is not a recognizable form and requires a Metropolis step. A candidate $\tilde{v}_{k}^{(t+1)}$ is drawn from a gamma density $G\left(r, r / v_{k}^{(t)}\right)$ with mean equal to the previous draw $v_{k}^{(t)}$ and is accepted with probability 


$$
\min \left\{1, \frac{p\left(\tilde{v}_{k}^{(t+1)} \mid \mathbf{x}, \mathbf{z}^{(t+1)}, \mathbf{w}^{(t+1)}, \boldsymbol{\mu}^{(t+1)}\right)}{p\left(v_{k}^{(t)} \mid \mathbf{x}, \mathbf{z}^{(t+1)}, \mathbf{w}^{(t+1)}, \boldsymbol{\mu}^{(t+1)}\right)} \frac{p\left(\tilde{v}_{k}^{(t+1)}, v_{k}^{(t)}\right)}{p\left(v_{k}^{(t)}, \tilde{v}_{k}^{(t+1)}\right)}\right\}
$$

where $p\left(v_{k}^{(t)}, \tilde{v}_{k}^{(t+1)}\right)$ is the gamma density used to generate $\tilde{v}_{k}^{(t+1)}$. The value of $r$ is chosen by experimentation to give an acceptance rate of approximately 0.25 to 0.4 .

\section{DOMINANCE ASSESSMENT WITH THE GAMMA MIXTURE}

Having obtained $M$ MCMC draws from the posterior distribution $p(\boldsymbol{\mu}, \mathbf{v}, \mathbf{w} \mid \mathbf{x})$, we can proceed to set up the machinery to assess various types of dominance. Associated with each draw $\left(\boldsymbol{\mu}^{(\boldsymbol{m})}, \mathbf{v}^{(\boldsymbol{m})}, \mathbf{w}^{(\boldsymbol{m})}\right), \quad m=1,2, \ldots, M \quad$, we have a gamma mixture density $p\left(x \mid \boldsymbol{\mu}^{(m)}, \mathbf{v}^{(m)}, \mathbf{w}^{(m)}\right)$ that can be obtained from (10). If interest centers on the predictive density for $x$, it can be obtained by averaging $p\left(x \mid \boldsymbol{\mu}^{(m)}, \mathbf{v}^{(m)}, \mathbf{w}^{(m)}\right)$ over all the parameter draws. However, for assessing dominance our interest centres on (1) the Lorenz curves $L_{X}\left(u \mid \boldsymbol{\mu}^{(m)}, \mathbf{v}^{(m)}, \mathbf{w}^{(m)}\right),(2)$ the generalized Lorenz curves

$$
G L_{X}\left(u \mid \mu^{(m)}, \mathbf{v}^{(m)}, \mathbf{w}^{(m)}\right)=\mu^{(m)} L_{X}\left(u \mid \mu^{(m)}, \mathbf{v}^{(m)}, \mathbf{w}^{(m)}\right)
$$

where $\mu^{(m)}=\sum_{k=1}^{K} w_{k}^{(m)} \mu_{k}^{(m)}$, and (3) the quantile functions $F^{-1}\left(u \mid \boldsymbol{\mu}^{(m)}, \mathbf{v}^{(m)}, \mathbf{w}^{(m)}\right)$, for each of the parameter draws $m=1,2, \ldots, M$.

The first challenge is to obtain a value of the quantile function $F^{-1}\left(u \mid \boldsymbol{\mu}^{(m)}, \mathbf{v}^{(m)}, \mathbf{w}^{(m)}\right)$ for a given proportion $u$, and a given set of parameters $\left(\boldsymbol{\mu}^{(m)}, \mathbf{v}^{(m)}, \mathbf{w}^{(m)}\right)$. Inverting the distribution function for a mixture of gamma densities is not straightforward. A closed form solution cannot be obtained, and solving the required nonlinear equation numerically for a large number of MCMC draws is not only time consuming, but also fraught with dangers of non-convergence. To overcome these problems we propose the following algorithm. 
1. Implement for each parameter draw $m=1,2, \ldots, M$.

2. Generate a large number of draws from $p\left(x \mid \boldsymbol{\mu}^{(m)}, \mathbf{v}^{(m)}, \mathbf{w}^{(m)}\right)$, say 100,000 , and sort them from lowest to highest. Let the vector of these draws be denoted by $\mathbf{x}$ and the $j$ th ordered value by $x_{j}$.

3. Find the 100,000 cumulative proportions $\boldsymbol{F}\left(\mathbf{x} \mid \boldsymbol{\mu}^{(m)}, \mathbf{v}^{(m)}, \mathbf{w}^{(m)}\right)$.

4. Implement for proportions $u_{i}, i=1,2, \ldots, n$.

5. For a given $u_{i}$, find the smallest $j$, call it $j_{i}$, for which $F\left(x_{j} \mid \boldsymbol{\mu}^{(m)}, \mathbf{v}^{(m)}, \mathbf{w}^{(m)}\right) \geq u_{i}$

6. Set a tolerable error $\varepsilon$. We used $\varepsilon=10^{-4}$ in our empirical work.

7. Find an initial value $\hat{y}_{1}=F^{-1}\left(u_{i} \mid \boldsymbol{\mu}^{(m)}, \mathbf{v}^{(m)}, \mathbf{w}^{(m)}\right)$ as a draw from the uniform distribution $U\left(x_{j_{i}-1}, x_{j_{i}}\right)$.

8. $\quad$ Compute $F_{X}\left(\hat{y}_{1} \mid \boldsymbol{\mu}^{(m)}, \mathbf{v}^{(m)}, \mathbf{w}^{(m)}\right)$. If $\left|F_{X}\left(\hat{y}_{1} \mid \boldsymbol{\mu}^{(m)}, \mathbf{v}^{(m)}, \mathbf{w}^{(m)}\right)-u_{i}\right|>\varepsilon$, go to step 9; otherwise, go back to 4. (Step 9 was seldom needed in our empirical work.)

9. We improve on the initial value $\hat{y}_{1}$ as follows. If $F_{X}\left(\hat{y}_{1} \mid \boldsymbol{\mu}^{(m)}, \mathbf{v}^{(m)}, \mathbf{w}^{(m)}\right)-u_{i}>0$, generate a random draw $\hat{y}_{2}=U\left(x_{j_{i}-1}, \hat{y}_{1}\right)$. If $F_{X}\left(\hat{y}_{1} \mid \boldsymbol{\mu}^{(m)}, \mathbf{v}^{(m)}, \mathbf{w}^{(m)}\right)-u_{i}<0$, generate $\hat{y}_{2}=U\left(\hat{y}_{1}, x_{j_{i}}\right)$.

10. Repeat step 8 with the new $\hat{y}$.

11. Go back to step 1 .

These values for the quantile function can be used to assess first-order stochastic dominance. For Lorenz and generalized Lorenz dominance we need to evaluate the first moment distribution function at the value for $\hat{y}$ computed from the above algorithm. 
Dropping the $(m)$ superscript for convenience, the first moment distribution function for the mixture of gamma densities is

$$
\begin{aligned}
F_{X}^{(1)}(\hat{y} \mid \boldsymbol{\mu}, \mathbf{v}, \mathbf{w}) & =\frac{1}{\mu} \int_{0}^{\hat{y}} x p(x \mid \mu, \mathbf{v}, \mathbf{w}) d x \\
& =\frac{1}{\mu} \sum_{k=1}^{K} w_{k} \int_{0}^{\hat{y}} x G\left(x \mid v_{k}, v_{k} / \mu_{k}\right) d x \\
& =\frac{1}{\mu} \sum_{k=1}^{K} w_{k} \int_{0}^{\hat{y}} \frac{\left(v_{k} / \mu_{k}\right)^{v_{k}}}{\Gamma\left(v_{k}\right)} x^{\left(v_{k}+1\right)-1} \exp \left(-\frac{v_{k} x}{\mu_{k}}\right) d x \\
& =\frac{1}{\mu} \sum_{k=1}^{K} w_{k} \int_{0}^{\hat{y}} \frac{\mu_{k}\left(v_{k} / \mu_{k}\right)^{v_{k}+1}}{\Gamma\left(v_{k}+1\right)} x^{\left(v_{k}+1\right)-1} \exp \left(-\frac{v_{k} x}{\mu_{k}}\right) d x \\
& =\frac{1}{\mu} \sum_{k=1}^{K} w_{k} \mu_{k} F_{k}\left(\hat{y} \mid v_{k}+1, v_{k} / \mu_{k}\right)
\end{aligned}
$$

where $F_{k}\left(\cdot \mid v_{k}+1, v_{k} / \mu_{k}\right)$ is the distribution function of a gamma density with parameters $\left(v_{k}+1\right)$ and $v_{k} / \mu_{k}$.

\section{DATA AND ESTIMATION RESULTS}

The data used to illustrate the methodology are household income data obtained for Indonesian urban regions for the years 1999, 2002, 2005, and 2008. These data were created using the household expenditure data obtained from the National Socio-Economic Survey. ${ }^{1}$ Appropriate price and equivalence-scale adjustments were made to ensure comparability over the years. Summary statistics for the data are presented in Table I. The units are thousands of rupiah per month.

If one makes judgments on the ordering of the distributions on the basis of the means or medians, the population becomes better off as it moves from 1999 to 2005 , but drops back in 2008, a likely consequence of the global financial crisis. Similarly, using the standard deviation and Gini coefficient to measure inequality suggests inequality increases from 1999

\footnotetext{
${ }^{1}$ The authors are grateful to Ari Handayani for providing the data used in Handayani (2013).
} 
to 2005 , but then decreases in 2008. Assuming a welfare function where mean income contributes positively and the standard deviation or Gini contributes negatively, it is not clear whether the distribution in any one year is preferable. Comparing the mean and median incomes, and checking the mean against the maximum values, reveals an extremely long right tail, with a small number of households with very high incomes. For example, in 2002 the mean income was 432, the maximum income was 24903 , and the proportion of households with incomes greater than 2000 was only 0.0074 .

Goodness-of-fit criteria were used to choose a suitable number of mixture components up to a maximum of five. The parameters for the prior densities were as follows. We set $\varphi_{k}=1$ for all $k$, implying a flat prior for the mixture weights on each component. For the exponential prior on scale parameters $v_{k}$ we set $\theta=0.01$ for all $k$. A $95 \%$ probability interval for this prior is $(2.54,202.86)$ implying a large range of values are possible. For the $\mu_{k}$ we set $\alpha_{k}=2.2$ for all $k$, and $\beta_{1}=60, \beta_{2}=100, \beta_{3}=150, \beta_{4}=200$ and $\beta_{5}=250$. The $95 \%$ probability intervals for $\mu_{1}, \mu_{2}, \ldots, \mu_{5}$ are $(10.31,202.86),(17.06,320.84),(24.93,488.68)$, $(33.85,626.13)$, and $(42.47,813.53)$, respectively. All these intervals suggest that the priors are relatively uninformative.

To assess goodness-of-fit for components $K=1,2 \ldots, 5$, we drew initial MCMC samples of 15000 , of which 5000 were discarded as a burn in. Maximum likelihood estimates for the Singh-Maddala and Dagum distributions were also obtained as a benchmark for comparison with the gamma mixtures. For each of the four years, three criteria were used to compare the estimated distribution functions $\widehat{F\left(x_{j}\right)}$, with the empirical distributions $F_{0}\left(x_{j}\right)=j / n$, where $j$ refers to the $j^{\text {th }}$ observation after ordering them from lowest to highest. The $\widehat{F\left(x_{j}\right)}$ were computed at the posterior means for the gamma mixture components and at the maximum 
likelihood estimates for the Singh-Maddala and Dagum distributions. The three goodness-offit criteria are

$$
\begin{gathered}
d=\max _{j}\left|\widehat{F\left(x_{j}\right)}-F_{0}\left(x_{j}\right)\right| \\
R M S E=\sqrt{\frac{1}{n} \sum_{i=1}^{n}\left(\widehat{F\left(x_{i}\right)}-F_{0}\left(x_{i}\right)\right)^{2}} \\
M A E=\frac{1}{n} \sum_{i=1}^{n}\left|\widehat{F\left(x_{i}\right)}-F_{0}\left(x_{i}\right)\right|
\end{gathered}
$$

The results in Table II provide unambiguous support for a gamma mixture with 4 components. It is superior to all other models for every measure of goodness of fit, and for all years. There is also strong support for the choice of a mixture model over the commonly used Singh-Maddala and Dagum distributions. We therefore chose the 4-component gamma mixture to obtain the draws used for dominance assessment in Section 6. We re-estimated the 4-component mixture with 50 different MCMC chains. For each chain, 11000 draws were generated, the first 1000 draws were discarded as a burn-in, and only every 50th draw of the subsequent 10000 draws was retained. With 50 chains and 200 retained observations from each chain, a total of $M=10000$ independent draws were retained for subsequent analysis. All parameters showed evidence of converging. A relatively small number of independent MCMC draws was chosen in preference to a large-number of correlated draws to reduce computing time for inverting the distribution functions and carrying out the dominance comparisons. The estimated densities for the 4-component mixtures are presented together with corresponding histograms in Figures 1, 2, 3, and 4. The shape of each distribution has been captured well by the 4-component mixtures, confirming the ability of the mixture distribution to capture the essential features of each distribution. Posterior means and standard deviations for each of the parameters for all 
components and all years are given in Table III. In general, the weight for the last component is very small.

\section{DOMINANCE RESULTS}

We begin a discussion of the dominance results by visually comparing the estimated distribution functions (Figure 5), the generalized Lorenz curves (Figure 6), and the Lorenz curves (Figure 7) for each of the 4 years. In Figure 5, it appears that the distributions in 2002, 2005 and 2008 all FSD the 1999 distribution. However, it seems that there is no clear ranking between 2002, 2005, and 2008. In Figure 6 the generalized Lorenz curves for 2002, 2005, and 2008 are also everywhere above the 1999 distribution, consistent with the fact that FSD implies GLD. A similar but less clear remark can be made about the Lorenz curves in Figure 7. Here the 1999 distribution Lorenz dominates 2002, 2005, and 2008, except for grid points where $u$ is close to zero or one.

When comparing the four years of income distributions, there are six possible pairwise comparisons that can be made. For each comparison, we calculate the probability that $X$ dominates $Y$, the probability that $Y$ dominates $X$, and the corresponding upper and lower bounds for the estimated probabilities. These probabilities are reported in Table IV, along with bounds computed using $C=1000$. The values in the table can be used to determine the probability that neither $X$ nor $Y$ dominates, given by $1-\operatorname{Pr}\left(X \geq_{D} Y\right)-\operatorname{Pr}\left(Y \geq_{D} X\right)$. In each of the three segments in the table, the last two columns provide evidence of how the income distribution has changed from one period to the next. We consider each pair of years in turn.

For 1999 and 2002, the dominance probabilities in the table make clear predictions. In terms of FSD and GLD, the probability that 2002 dominates 1999 is greater than 0.99 . While for LD, the probability that 1999 dominates 2002 is 0.9996 . The level of welfare measured by FSD and the level of relative inequality described by LD have increased significantly from 1999 to 2002. With respect to GLD, we can conclude that growth over the period has been 
sufficiently large enough to compensate for the increase in inequality, making social welfare unambiguously greater in 2002. In contrast, when comparing 2002 and 2005, we find the probability that neither year is dominant is very close to 1 for FSD, GLD and LD. There is a only very low probability (0.0037) that 2002 dominates 2005 in terms of LD. We obtain a similar result for FSD and GLD when comparing 2005 to 2008; the probability that neither year is dominant is very close to 1 . However, in terms of LD, the probability that 2008 dominates 2005 is close to 1 . There are also positive probabilities $(0.9996,0.1915$, and 0.3331) that 1999 Lorenz dominates 2002, 2005, and 2008, respectively. These results lead us to conclude that the level of inequality has increased from 1999 to 2005 , and decreased from 2005 to 2008. The only clear improvement in terms of FSD and GLD is from 1999 to 2002 (0.9990), and 1999 to 2008 (0.9997). It is somewhat surprising that the FSD and GLD probabilities of 2005 over 1999 ( 0.2529 and 0.4676, respectively) are far less than those for 2002 over 1999 (both over 0.99). Since both the mean and median for 2005 are greater than those for 2002, we might expect the FSD and GLD probabilities for 2005 over 1999 to be greater than those for 2002 over 1999. Another surprising result comes from comparing the Lorenz dominance probabilities and the estimated Gini coefficients for 2002, 2005 and 2008. The Gini coefficient for 2005 (0.3797) is higher than those for both $2002(0.3509)$ and 2008 (0.3583). Hence, we may expect similar LD probabilities for 2008 over 2005, and 2002 over 2005. However, the probability 2008 Lorenz dominates 2005 is 1, while the probability 2002 Lorenz dominates 2005 is only 0.0037 . These examples illustrate the usefulness of using posterior probabilities in addition to summary statistics from the distributions. They provide us with some evidence that there may be subtle changes occurring in different parts of the distribution over time that are not captured by the summary statistics. These aspects can be investigated further by using probability curves to assess the probability of dominance at different sub-intervals of the population proportions. 
Recall that the probability curves provide us with the probability of dominance at each population proportion $u$. Therefore we can use these curves to see which population proportions have the greatest effect on the probability of dominance, or lack of it. Furthermore we are able to show how the probability of dominance will change if we restrict our focus to a particular segment of the population, such as the poorest $10 \%$ or $20 \%$. The probability of dominance within the restricted range will necessarily be less than or equal to the minimum value of the probability curve for that restricted range. Additionally, the probability curve for dominance in one direction is the mirror image of the probability curve of dominance in the other direction. For example, $\operatorname{Pr}\left(2002 \geq_{F S D} 1999 \mid u\right)$ is the mirror image of $\operatorname{Pr}\left(1999 \geq_{F S D} 2002 \mid u\right)$.

The probability curves for 2005 over 1999 are given in Figure 8. The lower and upper bounds of the probability curves are also included but are visually indistinguishable from the mean curve. By observing Figure 8 we can see that the FSD and GLD probabilities for 2005 over 1999 are very sensitive to the starting point of the population proportion $u$. If we restrict our attention to $u \geq 0.005$, then, instead of being only 0.25 and 0.47 , both dominance probabilities are greater than 0.95 , bringing them much in line with the FSD and GLD probabilities for 2002 over 1999. Thus, the probability curves enable us to isolate the cause of significant differences between the dominance probabilities. Between 2002 and 2005 the incomes of the poorest $0.5 \%$ of the sample must have declined sufficiently such that we are unable to establish either a FSD or GLD relationship for 2005 over 1999. Such a precise statement cannot be made using only the summary statistics.

To identify the cause of the other surprising result, we can view the probability curves for 2002 over 2005 and 2008 over 2005, given in Figures 9 and 10, respectively. If we restrict the analysis to approximately $u<0.98$, instead of being only 0.0037 , the probability that 2002 Lorenz dominates 2005 is greater than 0.9 , a similar LD probability to 
2008 over 2005 when unrestricted. We can use this information to infer several interesting things. The richest $2 \%$ of the sample in 2002 must be sufficiently richer than the richest $2 \%$ in 2008 to prevent us from making a definitive statement about how income inequality changed from 2002 to 2005. In other words, we cannot establish a Lorenz dominance relationship for 2002 over 2005 only because of the level of incomes of the richest $2 \%$. This captures an important aspect of the distributions that we could not establish simply through the use of Gini coefficients as summary statistics. It is, however, consistent with another summary statistic; the maximum income for $2002(24,903)$ is almost double that of 2008 $(13,182)$.

This more thorough investigation of what initially were surprising results demonstrates the usefulness of examining more than simple summary statistics when making statements about welfare changes. The probability curves help reveal the complete picture of changes in the income distribution. Also, the example underscores the importance of precisely estimating the quantile functions close to the bounds. This was a significant issue encountered in Chotikapanich and Griffiths (2006) when using the Singh-Maddala and Dagum distributions.

The probability curves are not only useful for isolating population proportions that are critical for dominance assessment. They can also be used to investigate dominance over restricted regions. Poverty orderings, for example, are concerned with dominance below a poverty line. A dominance probability within a restricted range is only likely to differ from a corresponding unrestricted dominance probability if the minimum of the probability curve occurs outside the restricted region. Moreover, the probability curve shows how the restricted dominance probability is likely to change as the restricted interval (for example, the poverty line) changes. 
Table $\mathrm{V}$ contains dominance results for the lowest $10 \%$ of the population. Comparing the unrestricted and restricted probabilities of dominance for 2002 over 2005 illustrates how striking the difference can be. The unrestricted probabilities did not allow us to make any statements about how welfare differs across years, since the probability of FSD, GLD and LD for 2002 over 2005 were 0, 0, and 0.0037, respectively. After restricting the range, however, these probabilities increased to $0.5330,0.9754$, and 1 , respectively. Examining the probability curves for FSD and GLD in Figure 9 reveals that restricting the range to less than $10 \%$ would further increase the probability of dominance. Similar remarks can be made for GLD dominance of 2008 over 2005 where the probability increased from 0.000 to 0.591 ; see Figure 10 .

\subsection{Comparison of Bayesian and Sampling Theory Results}

It is instructive to compare the Bayesian posterior probabilities with some sampling theory results and highlight any differences that may emerge. In Table V1 we report the probabilities of dominance alongside the $p$-values obtained using the Barrett and Donald (2003) tests for FSD and GLD and the Barrett et al. (2014) test for LD. The p-values are those obtained assuming a null hypothesis of dominance is true. In terms of the general conclusions about dominance that are likely to be made from each of the approaches, there is agreement in most cases. However, there are some glaring differences that deserve closer investigation.

Comparing 2005 and 2008, we find that, for FSD and GLD, the sampling theory $p$ values (0.8907 and 0.7610 for 2005 dominating 2008 and 0.0011 and 0.0000 for 2008 dominating 2005) would lead us to conclude that 2005 dominates 2008. However, the 
Bayesian approach gives a probability of 0.9999 that neither year dominates. ${ }^{2}$ Examining the probability curves in Figure 10, we find that the Bayesian result is largely driven by the poorest segment of the population. For FSD, ignoring the poorest $10 \%$ would give a dominance probability for 2005 over 2008 of approximately 0.5 . Ignoring the poorest $10 \%$ and the middle $25-60 \%$ would increase this probability to 1 . For GLD, ignoring the poorest $20 \%$ would give a dominance probability of approximately 0.85 . A similar outcome occurs when examining FSD and GLD for 2008 and 2002, and 2005 and 2002. The Bayesian probabilities that neither dominates are almost 1, whereas the sampling theory $p$-values of 0.4264 and 0.4625 for FSD and 0.6100 and 06880 for SSD, along with zero values in the other direction, suggest that 2002 is dominated by both 2005 and 2008 . Taking 2002 and 2005 as an example, the probability curves in Figure 9 suggest that the probability of 2005 dominating 2002 is 1 if we ignore the poorest $16 \%$ of the population for FSD and the poorest $36 \%$ for GLD. It is perhaps dangerous to make a general conclusion from just these few examples, but it appears that the sampling theory tests are likely to suggest dominance if there is a large range of population segments where the probability of dominance is 1 , even if there are some limited ranges where the probability is close to 0 .

The same issue can arise with LD. The p-values of 1 for 1999 dominating 2005 and 0 for 2005 dominating 1999 suggest 1999 is dominant, whereas the Bayesian probability that neither dominates is 0.8085 . The probability curve in Figure 8 shows that it is the richest $10 \%$ of the population that caused the discrepancy. Ignoring them would increase the Bayesian probability of dominance to 1.

\footnotetext{
${ }^{2}$ One should resist the temptation to treat the $p$-values as sampling theory probabilities of dominance. The $p$ value for GLD of 2005 over 2008 of 0.7610 is less than that for FSD which is 0.8907 . The probability of GLD must be at least as great as the probability of FSD.
} 


\section{CONCLUSIONS}

The development of statistical inference for assessing how income distributions have changed over time in what might be considered a desirable way has attracted a great deal of attention within the sampling theory framework. Hypothesis testing procedures have been developed for, among other things, Lorenz dominance, generalized Lorenz dominance and first-order stochastic dominance. This paper provides an alternative to the existing hypothesis tests, by defining a novel approach to assessing dominance relationships within a framework of Bayesian inference. A desirable feature of this framework is the reporting of results in terms of probabilities - a natural way to express our uncertainty. Furthermore, these probabilities can be provided for dominance in either direction, as well as the probability that dominance does not occur. They overcome the problem of giving favorable treatment to what is chosen as the null hypothesis in sampling theory inference. By employing a flexible gamma mixture model we minimize the sensitivity of dominance results to the chosen distribution. Additionally, we suggest an approach for calculating upper and lower bounds for the posterior probability of dominance, allowing inference to be made with greater confidence about the accuracy of the estimates. The methodology is applied to data for Indonesia for the years 1999, 2002, 2005, and 2008. In general, the dominance test results led us to conclude that the level of inequality increased from 1999 to 2005 , and decreased from 2005 to 2008 . There was also strong evidence of an increase in welfare from 1999 to 2002, but little evidence of improvements from 2002 to 2005 and from 2005 2008. The introduction of probability curves that trace how the probability of dominance at a particular population proportion changes as the population proportion changes enabled us to isolate segments of the population having the greatest impact on overall dominance and to explain seemingly contradictory outcomes from the Bayesian and sampling theory approaches. Future 
research will extend the framework to multivariate frameworks and to orderings of more than two distributions. 


\section{REFERENCES}

Anderson, G. (1996): Nonparametric Tests for Stochastic Dominance. Econometrica, 64, $1183-1193$

Atkinson, A. (1970): On the Measurement of Inequality. Journal of Economic Theory, 2, $244-263$

Atkinson, A. and Bourguignon, F. (1982): The Comparison of Multi-Dimensioned Distributions of Economic Status. Review of Economic Studies, 49, 183-201.

Bai, Z., Hua L., Liu, H. and Wong, W-K. (2011): Test Statistics for Prospect and Markowitz Stochastic Dominances with Applications. Econometrics Journal, 14, 278-303.

Barrett, G. F. and Donald, S. G. (2003): Consistent Tests for Stochastic Dominance. Econometrica, 71, (1), 71-104.

Barrett, G. F., Donald, S. G., and Bhattacharya D. (2014): Consistent Non-parametric Tests for Lorenz Dominance. Journal of Business and Economics Statistics, 32, (1), 1-13.

Barrett, G. F., Donald, S. G., and Hsu,Y.-C. (2016): Consistent Tests for Poverty Dominance Relations. Journal of Econometrics, 191, 360-373.

Beach, C.M., and Davidson R. (1983): Distribution-Free Statistical Inferences with Lorenz Curves and Income Shares, Review of Economic Studies, 50, 723-735.

Bennett, C.J. (2013): Inference for Dominance Relations. International Economic Review, $54,1309-1328$.

Bennett, C.J., and Mitra, S. (2016): Multidimensional Poverty: Measurement, Estimation and Inference, Econometric Reviews, 32, 57-83.

Berrendero, J.C., and Cárcamo, J. (2011): Tests for Second Order Stochastic Dominance Based on L-Statistics, Journal of Business and Economic Statistics, 29, 260-270. 
Bishop, J.A., Chakraborti, S., and Thistle, P.D. (1989): Asymptotically Distribution-Free Statistical Inferences for Generalized Lorenz Curves. Review of Economics and Statistics, 71, 725-727.

Bishop, J.A., Formby, J.P., and Sakano, R. (1995): Lorenz and Stochastic Dominance Comparisons of European Income Distributions, Research on Economic Inequality, 6, $77-92$.

Bishop, J.A., Formby, J.P., and Smith, W.J. (1991): Lorenz Dominance and Welfare: Changes in the U.S. Distribution of Income, 1967-1986, Review of Economics and Statistics, 73, 134-139.

Chotikapanich, D. and Griffiths, W. E. (2006): Bayesian Assessment of Lorenz and Stochastic Dominance in Income Distributions. Research on Economic Inequality, 13, $297-321$.

Dardanoni, V, and Forcina, A. (1999): Inference for Lorenz Curve Orderings. Econometrics Journal, 2, 49-75.

Davidson, R. and Duclos, J. Y. (2000): Statistical Inference for Stochastic Dominance and for the Measurement of Poverty and Inequality. Econometrica, 68, (6), 1435-1464.

Davidson, R. and Duclos, J. Y. (2013): Testing for Restricted Stochastic Dominance. Econometric Reviews, 32, 84-125.

Denuit, M., Dhaene, J., Goovaerts, M. J., and Kaas, R. (2005): Actuarial Theory for Dependent Risks: Measures, Orders, and Models. Wiley, New York

Donald, S. and Hsu,Y.-C. (2016): Improving the Power of Tests of Stochastic Dominance, Econometric Reviews, 35, 553-585.

Duclos, J. Y., Sahn, D., and Younger, S. (2006): Robust Multidimensional Poverty Comparisons. The Economic Journal, 116 (514), 943-968. 
Foster, J. E. and Shorrocks, A. F. (1988): Poverty Orderings and Welfare Dominance. Social Choice and Welfare, 5, 179-198.

Geweke, J. (1986): Exact Inference in the Inequality Constrained Normal Linear Regression Model. Journal of Applied Econometrics, 1, 127-142.

Geweke, J. (1988): The Secular and Cyclical Behavior of Real GDP in 19 OECD Countries, 1957-1983. Journal of Business and Economic Statistics, 6, 479-486.

Geweke, J. (2007): Interpretation and Inference in Mixture Models: Simple MCMC Works. Computational Statistics and Data Analysis, 51, 3529-3550.

Handayani, A. (2013): An Analysis of Changes in Indonesia's Income Distribution 19962008, PhD thesis, Monash University, Melbourne.

Horváth, L., Kokoszka, P. and Zitikis, R. (2006): Testing for Stochastic Dominance using the Weighted McFadden-Type Statistic. Journal of Econometrics, 133, 191-205.

Jenkins, S.P., and Lambert, P.J. (1997): Three 'L's of Poverty Curves, with an Analysis of UK Poverty Trends. Oxford Economic Papers, 49,317-327.

Kaas, R., Van Heerwaarden, A. E., and Goovaert, M. J. (1994): Orderings of Actuarial Risks. Caire, Brussels.

Kaur, A., Rao, P. B. L. S., and Singh, H. (1994): Testing for Second-Order Stochastic Dominance of Two Distributions. Econometric Theory, 10, 849-866

Kleiber, C. and Kotz, S. (2003): Statistical Size Distributions in Economics and Actuarial Sciences. New Jersey: Wiley and Sons.

Lambert, P. J. (2001): The Distribution and Redistribution of Income: A Mathematical Analysis. Third edition, Manchester University Press.

Linton, O., Maasoumi, E., and Whang, Y.-J. (2005): Consistent Testing for Stochastic Dominance under General Sampling Schemes. Review of Economic Studies, 72, 735765. 
Linton, O., Song, K., and Whang, Y.-J. (2010): An Improved Bootstrap Test for Stochastic Dominance. Journal of Econometrics, 154, 186-202.

Maasoumi, E. (1997): Empirical Analyses of Inequality and Welfare. In M.H. Pesaran and P. Schmidt (eds), Handbook of Applied Econometrics: Volume II Microeconomics, Blackwell, Malden, MA.

Maasoumi, E. and Heshmati, A. (2000): Stochastic Dominance among Swedish Income Distributions. Econometric Reviews, 19, 287-320.

Maasoumi, E. and Heshmati, A. (2008): Evaluating Dominance Ranking of PSID Incomes by Various Household Attributes. In G. Betti and A. Lemmi, Advances on Income Inequality and Concentration Measures, Routledge, Oxford.

Maasoumi, E. and Millimet (2005): Robust Inference Concerning Recent Trends in US Environmental Quality. Journal of Applied Econometrics, 20, 55-78.

McCaig, B., and Yatchew, A. (2007): International Welfare Comparisons and Nonparametric Testing of Multivariate Stochastic Dominance. Journal of Applied Econometrics, 22, 951-969.

McFadden, D. (1989): Testing for Stochastic Dominance, in studies in the Economics of Uncertainty: In Honor of Josef Hadar, ed. by Fomby, T. B. and Seo, T. K. New York, Berlin, London, Tokyo: Springer.

Schluter, C., and Trede M. (2002): Tails of Lorenz Curves. Journal of Econometrics, 109, 151-166.

Shorrocks, A. F. (1983): Ranking Income Distributions. Economica, 50, (197), 3-17.

Sriboonchitta, S., Wong, W. K., Dhompongsa, S., and Nguyen, H.T. (2009): Stochastic Dominance and Applications to Finance, Risk, and Economics. Chapman \& Hall/CRC, Boca Raton, Florida. 
Tabri, R.V. (2015): Empirical Likelihood for Robust Poverty Comparisons, Department of Economics, University of Sydney Working Paper 2015-2, http://econwpseries.com/2015/201502-5.pdf.

Wiper, M., Insua, D. R. and Ruggeri, F. (2001): Mixtures of Gamma Distributions with Applications. Journal of Computational and Graphical Statistics, 10, 440-454.

Wong, W. K., Phoon, K. F., and Lean, H. H. (2008): Stochastic Dominance Analysis of Asian Hedge Funds. Pacific Basin Finance Journal , 16, (3), 204-223. 
Table I: Sample Statistics (in Rp '000 per month)

\begin{tabular}{lrrrr}
\hline & 1999 & 2002 & 2005 & 2008 \\
\hline Sample mean & 332.63 & 432.36 & 477.04 & 454.08 \\
Median & 270.40 & 337.80 & 357.95 & 355.27 \\
Minimum & 44.12 & 57.65 & 38.32 & 59.84 \\
Maximum & 5973.92 & $24,902.67$ & $30,216.51$ & $13,181.99$ \\
Standard deviation & 249.32 & 477.29 & 511.19 & 401.23 \\
Gini Coefficient & 0.3184 & 0.3509 & 0.3797 & 0.3583 \\
Sample size & 25,175 & 29280 & 24,687 & 26,648 \\
Proportion more than 2000 & 0.00242 & 0.0074 & 0.0134 & 0.0099 \\
\hline
\end{tabular}


Table II: Goodness-of-fit comparisons

\begin{tabular}{|c|c|c|c|c|c|c|c|}
\hline & \multicolumn{7}{|c|}{1999} \\
\hline & $K=1$ & $K=2$ & $K=3$ & $K=4$ & $K=5$ & Dagum & S-M \\
\hline RMSE & 0.0478 & 0.0140 & 0.0059 & 0.0037 & 0.0080 & 0.0084 & 0.0125 \\
\hline$M A E$ & 0.0414 & 0.0120 & 0.0050 & 0.0030 & 0.0078 & 0.0076 & 0.0111 \\
\hline \multirow[t]{2}{*}{$d$} & 0.0766 & 0.0258 & 0.0199 & 0.0083 & 0.0119 & 0.0130 & 0.0197 \\
\hline & \multicolumn{7}{|c|}{2002} \\
\hline$R M S E$ & 0.0559 & 0.0189 & 0.0065 & 0.0046 & 0.0086 & 0.0079 & 0.0117 \\
\hline$M A E$ & 0.0491 & 0.0157 & 0.0055 & 0.0037 & 0.0081 & 0.0069 & 0.0103 \\
\hline \multirow[t]{2}{*}{$d$} & 0.0853 & 0.0333 & 0.0116 & 0.0107 & 0.0131 & 0.0128 & 0.0198 \\
\hline & \multicolumn{7}{|c|}{2005} \\
\hline RMSE & 0.0556 & 0.0172 & 0.0046 & 0.0023 & 0.0086 & 0.0079 & 0.0122 \\
\hline$M A E$ & 0.0488 & 0.0145 & 0.0040 & 0.0018 & 0.0081 & 0.0067 & 0.0106 \\
\hline \multirow[t]{2}{*}{$d$} & 0.0864 & 0.0325 & 0.0093 & 0.0062 & 0.0132 & 0.0151 & 0.0209 \\
\hline & \multicolumn{7}{|c|}{2008} \\
\hline$R M S E$ & 0.0472 & 0.0156 & 0.0047 & 0.0026 & 0.0034 & 0.0112 & 0.0133 \\
\hline$M A E$ & 0.0414 & 0.0134 & 0.0041 & 0.0021 & 0.0030 & 0.0097 & 0.0114 \\
\hline$d$ & 0.0726 & 0.0269 & 0.0086 & 0.0064 & 0.0068 & 0.0191 & 0.0224 \\
\hline
\end{tabular}


Table III: Posterior means and standard deviations (in parenthesis) of mixture parameter estimates and mixture weights

\begin{tabular}{|c|c|c|c|c|c|}
\hline & & \multicolumn{4}{|c|}{ Component } \\
\hline & & 1 & 2 & 3 & 4 \\
\hline \multirow[t]{3}{*}{1999} & $\mu$ & $\begin{array}{r}176.5033 \\
(4.8098)\end{array}$ & $\begin{array}{l}294.4166 \\
(10.2637)\end{array}$ & $\begin{array}{l}515.4174 \\
(25.5746)\end{array}$ & $\begin{array}{l}1296.5664 \\
(138.7104)\end{array}$ \\
\hline & $w$ & $\begin{array}{r}0.2497 \\
(0.0366)\end{array}$ & $\begin{array}{r}0.4976 \\
(0.0336)\end{array}$ & $\begin{array}{r}0.2361 \\
(0.0332)\end{array}$ & $\begin{array}{r}0.0166 \\
(0.0036)\end{array}$ \\
\hline & $v$ & $\begin{array}{l}13.9886 \\
(1.1937)\end{array}$ & $\begin{array}{r}8.1546 \\
(0.5342)\end{array}$ & $\begin{array}{r}5.0413 \\
(0.4295)\end{array}$ & $\begin{array}{r}2.1930 \\
(0.3500)\end{array}$ \\
\hline \multirow[t]{3}{*}{2002} & $\mu$ & $\begin{array}{r}234.8722 \\
(3.8026)\end{array}$ & $\begin{array}{r}430.4595 \\
(8.7793)\end{array}$ & $\begin{array}{l}918.7395 \\
(39.9731)\end{array}$ & $\begin{array}{r}4141.7860 \\
(637.1302)\end{array}$ \\
\hline & $w$ & $\begin{array}{r}0.3466 \\
(0.0256)\end{array}$ & $\begin{array}{r}0.5383 \\
(0.0245)\end{array}$ & $\begin{array}{r}0.1105 \\
(0.0111)\end{array}$ & $\begin{array}{r}0.0045 \\
(0.0009)\end{array}$ \\
\hline & $v$ & $\begin{array}{r}9.9992 \\
(0.4361)\end{array}$ & $\begin{array}{r}6.1073 \\
(0.2747)\end{array}$ & $\begin{array}{r}3.4621 \\
(0.2771)\end{array}$ & $\begin{array}{r}1.2346 \\
(0.2343)\end{array}$ \\
\hline \multirow[t]{3}{*}{2005} & $\mu$ & $\begin{array}{r}232.9290 \\
(4.5749)\end{array}$ & $\begin{array}{r}441.1039 \\
(11.6864)\end{array}$ & $\begin{array}{r}901.9546 \\
(57.0056)\end{array}$ & $\begin{array}{r}2557.9964 \\
(313.5324)\end{array}$ \\
\hline & $w$ & $\begin{array}{r}0.3073 \\
(0.0281)\end{array}$ & $\begin{array}{r}0.5277 \\
(0.0308)\end{array}$ & $\begin{array}{c}0.1492 \\
(0.0200)\end{array}$ & $\begin{array}{r}0.0157 \\
(0.0038)\end{array}$ \\
\hline & $v$ & $\begin{array}{r}9.2294 \\
(0.4858)\end{array}$ & $\begin{array}{r}5.5929 \\
(0.3738)\end{array}$ & $\begin{array}{r}3.3478 \\
(0.3371)\end{array}$ & $\begin{array}{r}1.5555 \\
(0.2293)\end{array}$ \\
\hline \multirow[t]{3}{*}{2008} & $\mu$ & $\begin{array}{r}198.3503 \\
(5.7395)\end{array}$ & $\begin{array}{l}361.0210 \\
(15.6557)\end{array}$ & $\begin{array}{l}625.4886 \\
(41.1883)\end{array}$ & $\begin{array}{r}1411.7252 \\
(95.0639)\end{array}$ \\
\hline & $w$ & $\begin{array}{r}0.2014 \\
(0.0264)\end{array}$ & $\begin{array}{r}0.4674 \\
(0.0424)\end{array}$ & $\begin{array}{r}0.2800 \\
(0.0468)\end{array}$ & $\begin{array}{r}0.0512 \\
(0.0079)\end{array}$ \\
\hline & $v$ & $\begin{array}{l}12.6651 \\
(0.9888)\end{array}$ & $\begin{array}{r}7.4202 \\
(0.5833)\end{array}$ & $\begin{array}{r}5.2249 \\
(0.5254)\end{array}$ & $\begin{array}{r}2.2095 \\
(0.1686)\end{array}$ \\
\hline
\end{tabular}


Table IV: Dominance probabilities, with bounds in parentheses

\begin{tabular}{|c|c|c|c|c|c|c|}
\hline & $08 \mathrm{D} 05$ & $05 \mathrm{D} 08$ & & & & \\
\hline FSD & $\begin{array}{c}0.0000 \\
(0.0000,0.0000)\end{array}$ & $\begin{array}{c}0.0001 \\
(0.0000,0.0004)\end{array}$ & & & & \\
\hline GLD & $\begin{array}{c}0.0000 \\
(0.0000,0.0000)\end{array}$ & $\begin{array}{c}0.0001 \\
(0.0000,0.0005)\end{array}$ & & & & \\
\hline \multirow[t]{2}{*}{ LD } & $\begin{array}{c}1.0000 \\
(0.9997,1.0000)\end{array}$ & $\begin{array}{c}0.0000 \\
(0.0000,0.0000)\end{array}$ & & & & \\
\hline & $08 \mathrm{D} 02$ & $02 \mathrm{D} 08$ & $05 \mathrm{D} 02$ & $02 \mathrm{D} 05$ & & \\
\hline FSD & $\begin{array}{c}0.0000 \\
(0.0000,0.0001)\end{array}$ & $\begin{array}{c}0.0000 \\
(0.0000,0.0000)\end{array}$ & $\begin{array}{c}0.0001 \\
(0.0000,0.0004)\end{array}$ & $\begin{array}{c}0.0000 \\
(0.0000,0.0000)\end{array}$ & & \\
\hline GLD & $\begin{array}{c}0.0148 \\
(0.0117,0.0183)\end{array}$ & $\begin{array}{c}0.0000 \\
(0.0000,0.0000)\end{array}$ & $\begin{array}{c}0.0002 \\
(0.0000,0.0006)\end{array}$ & $\begin{array}{c}0.0000 \\
(0.0000,00000)\end{array}$ & & \\
\hline \multirow[t]{2}{*}{ LD } & $\begin{array}{c}0.0000 \\
(0.0000,0.0000)\end{array}$ & $\begin{array}{c}0.0000 \\
(0.0000 .0000)\end{array}$ & $\begin{array}{c}0.0000 \\
(0.0000,0.0000)\end{array}$ & $\begin{array}{c}0.0037 \\
(0.0021,0.0056)\end{array}$ & & \\
\hline & 08 D 99 & 99 D 08 & 05 D 99 & 99 D 05 & 02 D 99 & 99 D 02 \\
\hline FSD & $\begin{array}{c}0.9980 \\
(0.9966,0.9991)\end{array}$ & $\begin{array}{c}0.0000 \\
(0.0000,0.0000)\end{array}$ & $\begin{array}{c}0.2529 \\
(0.2432,0.2624)\end{array}$ & $\begin{array}{c}0.0000 \\
(0.0000,0.0000)\end{array}$ & $\begin{array}{c}0.9943 \\
(0.9923,0.9962)\end{array}$ & $\begin{array}{c}0.0000 \\
(0.0000,0.0000)\end{array}$ \\
\hline GLD & $\begin{array}{c}0.9997 \\
(0.9989,1.0000)\end{array}$ & $\begin{array}{c}0.0000 \\
(0.0000,0.0000)\end{array}$ & $\begin{array}{c}0.4676 \\
(0.4595,0.4772)\end{array}$ & $\begin{array}{c}0.0000 \\
(0.0000,0.0000)\end{array}$ & $\begin{array}{c}0.9990 \\
(0.9979,0.9998)\end{array}$ & $\begin{array}{c}0.0000 \\
(0.0000,0.0000)\end{array}$ \\
\hline LD & $\begin{array}{c}0.0000 \\
(0.0000,0.0000)\end{array}$ & $\begin{array}{c}0.3331 \\
(0.3247,0.3421)\end{array}$ & $\begin{array}{c}0.0000 \\
(0.0000,0.0000)\end{array}$ & $\begin{array}{c}0.1915 \\
(0.1829,0.2020)\end{array}$ & $\begin{array}{c}0.0000 \\
(0.0000,0.0000)\end{array}$ & $\begin{array}{c}0.9996 \\
(0.9988,1.0000)\end{array}$ \\
\hline
\end{tabular}


Table V: Restricted dominance probabilities (for the lowest $10 \%$ of incomes), with bounds in parenthesis

\begin{tabular}{|c|c|c|c|c|c|c|}
\hline & $08 \mathrm{D} 05$ & $05 \mathrm{D} 08$ & & & & \\
\hline FSD & $\begin{array}{c}0.0108 \\
(0.0081,0.0136)\end{array}$ & $\begin{array}{c}0.0001 \\
(0.0000,0.0005)\end{array}$ & & & & \\
\hline GLD & $\begin{array}{c}0.5910 \\
(0.5796,0.6000)\end{array}$ & $\begin{array}{c}0.0001 \\
(0.0000,0.0005)\end{array}$ & & & & \\
\hline \multirow[t]{2}{*}{ LD } & $\begin{array}{c}1.0000 \\
(0.9999,1.0000)\end{array}$ & $\begin{array}{c}0.0000 \\
(0.0000,0.0000)\end{array}$ & & & & \\
\hline & $08 \mathrm{D} 02$ & $02 \mathrm{D} 08$ & $05 \mathrm{D} 02$ & $02 \mathrm{D} 05$ & & \\
\hline FSD & $\begin{array}{c}0.0027 \\
(0.0014,0.0042)\end{array}$ & $\begin{array}{c}0.1378 \\
(0.1306,0.1447)\end{array}$ & $\begin{array}{c}0.0002 \\
(0.0000,0.0006)\end{array}$ & $\begin{array}{c}0.5330 \\
(0.5250,0.5419)\end{array}$ & & \\
\hline GLD & $\begin{array}{c}0.0288 \\
(0.0240,0.0330)\end{array}$ & $\begin{array}{c}0.1297 \\
(0.1229,0.1360)\end{array}$ & $\begin{array}{c}0.0002 \\
(0.0000,0.0006)\end{array}$ & $\begin{array}{c}0.9754 \\
(0.9710,0.9788)\end{array}$ & & \\
\hline \multirow[t]{2}{*}{ LD } & $\begin{array}{c}0.0000 \\
(0.0000,0.0000)\end{array}$ & $\begin{array}{c}0.8426 \\
(0.8357,0.8506)\end{array}$ & $\begin{array}{c}0.0000 \\
(0.0000,0.0000)\end{array}$ & $\begin{array}{c}1.0000 \\
(1.0000,1.0000)\end{array}$ & & \\
\hline & 08 D 99 & 99 D 08 & 05 D 99 & $99 \mathrm{D} 05$ & 02 D 99 & 99 D 02 \\
\hline FSD & $\begin{array}{c}0.9980 \\
(0.9966,0.9991)\end{array}$ & $\begin{array}{c}0.0000 \\
(0.0000,0.0000)\end{array}$ & $\begin{array}{c}0.2529 \\
(0.2432,0.2624)\end{array}$ & $\begin{array}{c}0.0000 \\
(0.0000,0.0000)\end{array}$ & $\begin{array}{c}0.9943 \\
(0.9923,0.9962)\end{array}$ & $\begin{array}{c}0.0000 \\
(0.0000,0.0000)\end{array}$ \\
\hline GLD & $\begin{array}{c}0.9997 \\
(0.9989,1.000)\end{array}$ & $\begin{array}{c}0.0000 \\
(0.0000,0.0000)\end{array}$ & $\begin{array}{c}0.4676 \\
(0.4595,0.4772)\end{array}$ & $\begin{array}{c}0.0000 \\
(0.0000,0.0000)\end{array}$ & $\begin{array}{c}0.9999 \\
(0.9979,0.9998)\end{array}$ & $\begin{array}{c}0.0000 \\
(0.0000,0.0000)\end{array}$ \\
\hline LD & $\begin{array}{c}0.0000 \\
(0.0000,0.0000)\end{array}$ & $\begin{array}{c}1.0000 \\
(1.0000,1.0000)\end{array}$ & $\begin{array}{c}0.0000 \\
(0.0000,0.0000)\end{array}$ & $\begin{array}{c}1.0000 \\
(1.0000,1.0000)\end{array}$ & $\begin{array}{c}0.0000 \\
(0.0000,0.0000)\end{array}$ & $\begin{array}{c}1.0000 \\
(1.0000,1.0000)\end{array}$ \\
\hline
\end{tabular}


Table VI: A comparison of sampling theory $p$-values with stochastic dominance probabilities

\begin{tabular}{|c|c|c|c|c|c|c|c|}
\hline & & 08 D 05 & 05 D 08 & & & & \\
\hline \multirow[t]{2}{*}{ FSD } & probability & 0.0000 & 0.0001 & & & & \\
\hline & $p$-value & 0.0011 & 0.8907 & & & & \\
\hline \multirow[t]{2}{*}{ GLD } & probability & 0.0000 & 0.0001 & & & & \\
\hline & $p$-value & 0.0000 & 0.7610 & & & & \\
\hline \multirow[t]{3}{*}{ LD } & probability & 1.0000 & 0.0000 & & & & \\
\hline & $p$-value & 1.0000 & 0.0000 & & & & \\
\hline & & 08 D 02 & 02 D 08 & $05 \mathrm{D} 02$ & $02 \mathrm{D} 05$ & & \\
\hline \multirow[t]{2}{*}{ FSD } & probability & 0.0000 & 0.0000 & 0.0001 & 0.0000 & & \\
\hline & $p$-value & 0.4264 & 0.0000 & 0.4625 & 0.0000 & & \\
\hline \multirow[t]{2}{*}{ GLD } & probability & 0.0148 & 0.0000 & 0.0002 & 0.0000 & & \\
\hline & $p$-value & 0.6100 & 0.0000 & 0.6480 & 0.0000 & & \\
\hline \multirow[t]{3}{*}{ LD } & probability & 0.0000 & 0.0000 & 0.0000 & 0.0037 & & \\
\hline & $p$-value & 0.0930 & 0.0090 & 0.0000 & 0.2590 & & \\
\hline & & 08 D 99 & 99 D 08 & 05 D 99 & 99 D 05 & 02 D 99 & $99 \mathrm{D} 02$ \\
\hline \multirow[t]{2}{*}{ FSD } & probability & 0.9980 & 0.0000 & 0.2529 & 0.0000 & 0.9943 & 0.0000 \\
\hline & $p$-value & 0.9713 & 0.0000 & 0.9988 & 0.0000 & 0.9580 & 0.0000 \\
\hline \multirow[t]{2}{*}{ GLD } & probability & 0.9997 & 0.0000 & 0.4676 & 0.0000 & 0.9990 & 0.0000 \\
\hline & $p$-value & 1.0000 & 0.0000 & 0.8590 & 0.0000 & 0.9990 & 0.0000 \\
\hline \multirow[t]{2}{*}{ LD } & probability & 0.0000 & 0.3331 & 0.0000 & 0.1915 & 0.0000 & 0.9996 \\
\hline & $p$-value & 0.0000 & 0.9970 & 0.0000 & 1.0000 & 0.0000 & 0.9940 \\
\hline
\end{tabular}


Figure 1: Histogram and estimated density with parameters set at posterior means for 1999

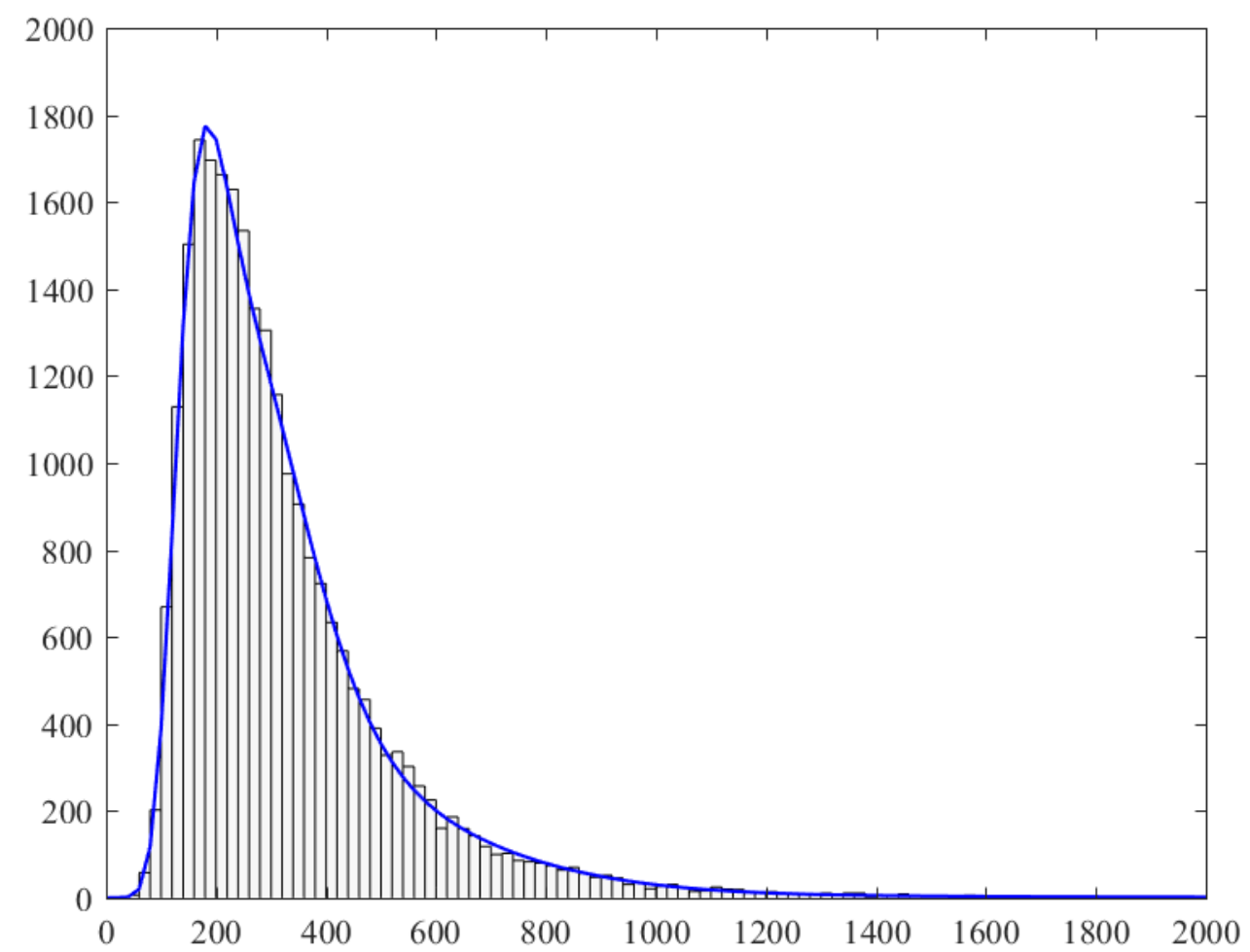

Figure 2: Histogram and estimated density with parameters set at posterior means for 2002

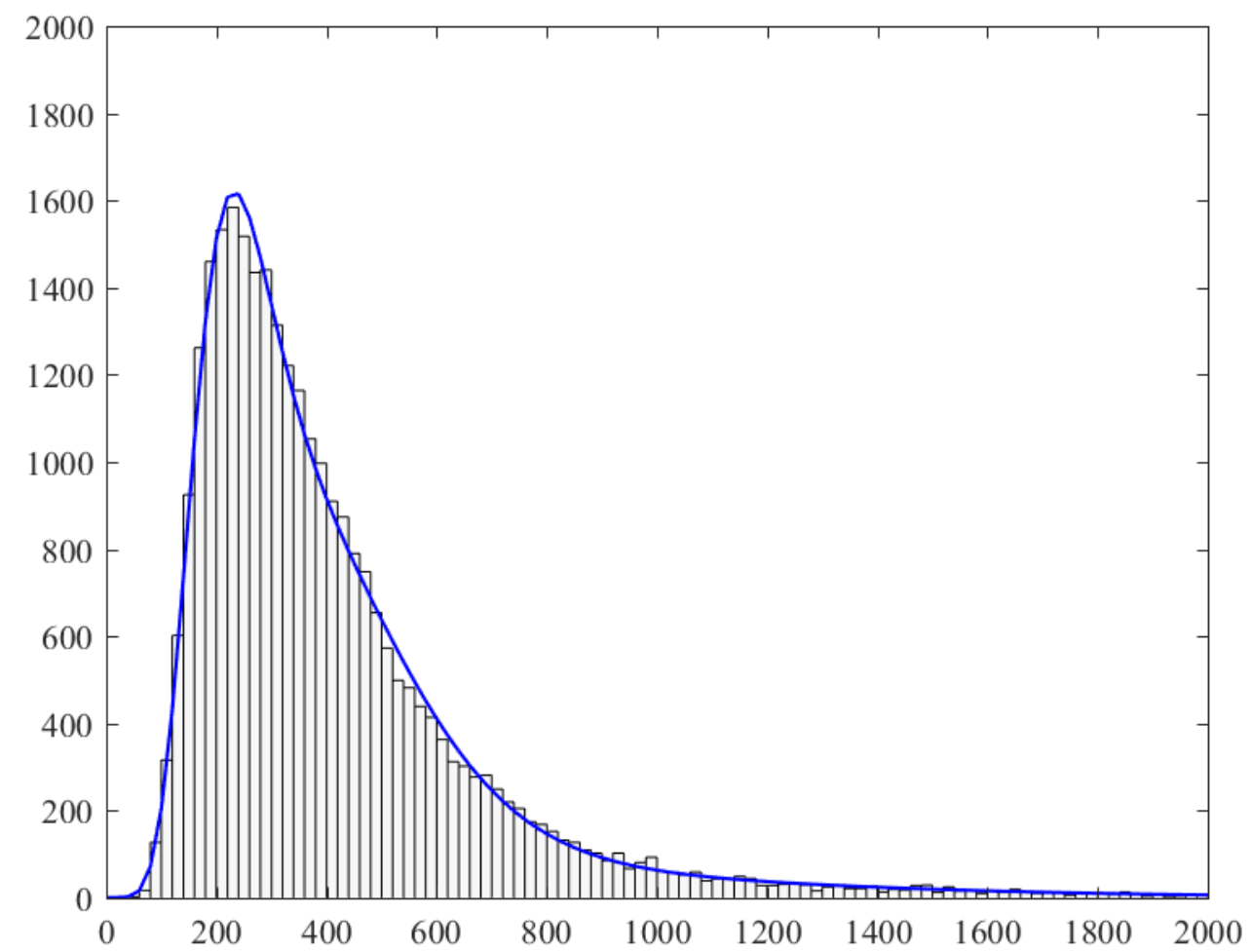


Figure 3: Histogram and estimated density with parameters set at posterior means for 2005

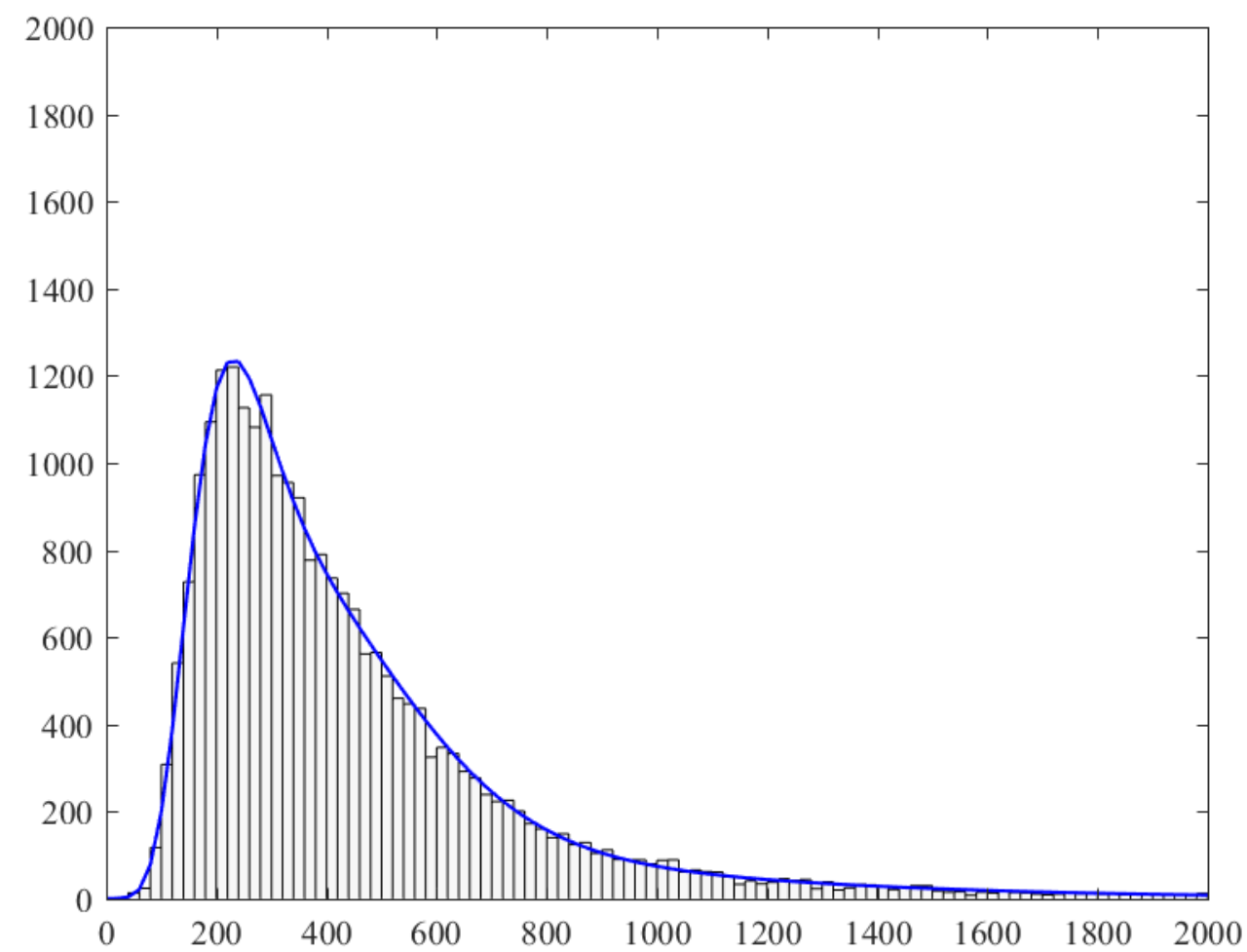

Figure 4: Histogram and estimated density with parameters set at posterior means for 2008

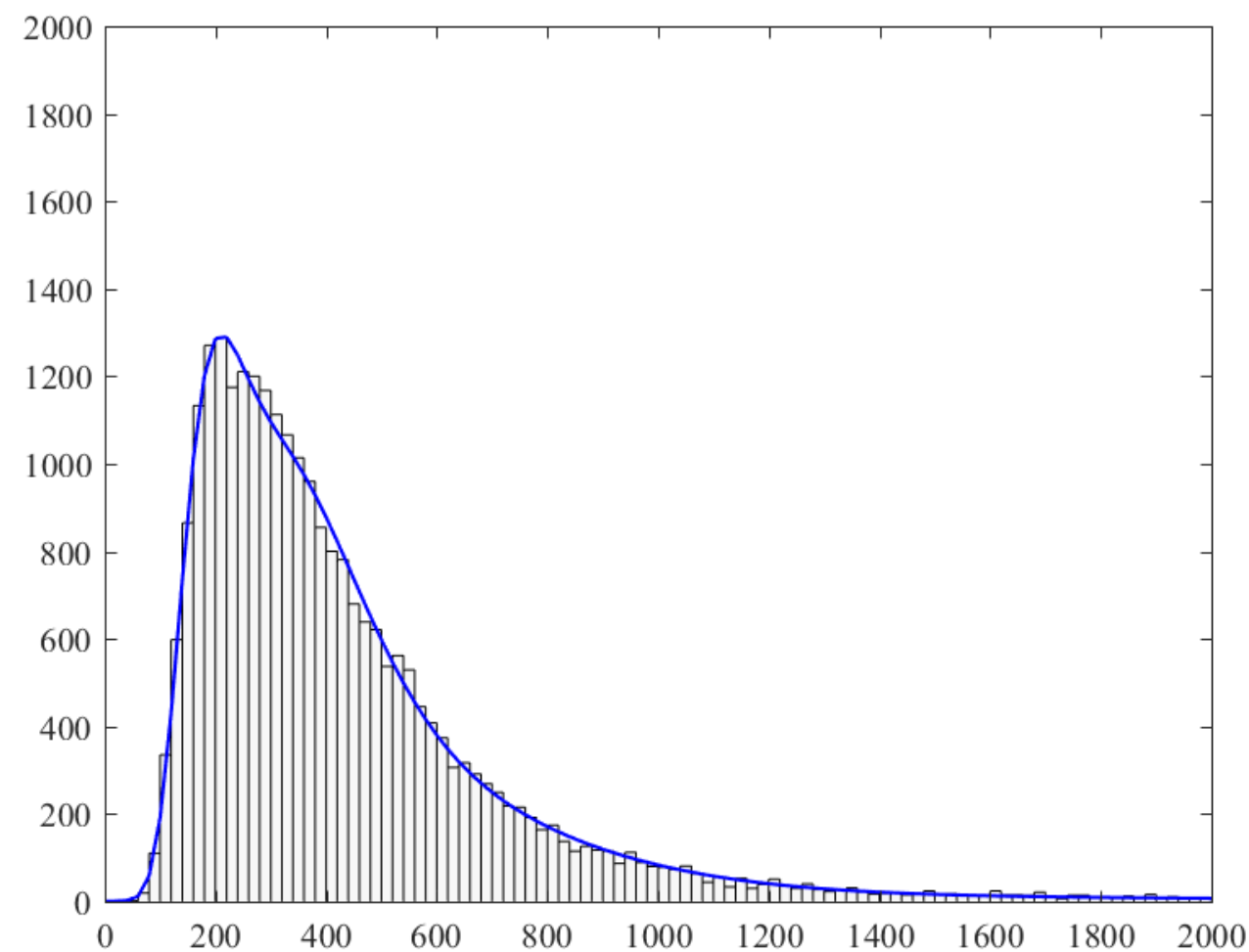


Figure 5: Estimated distribution functions with parameters set at posterior means

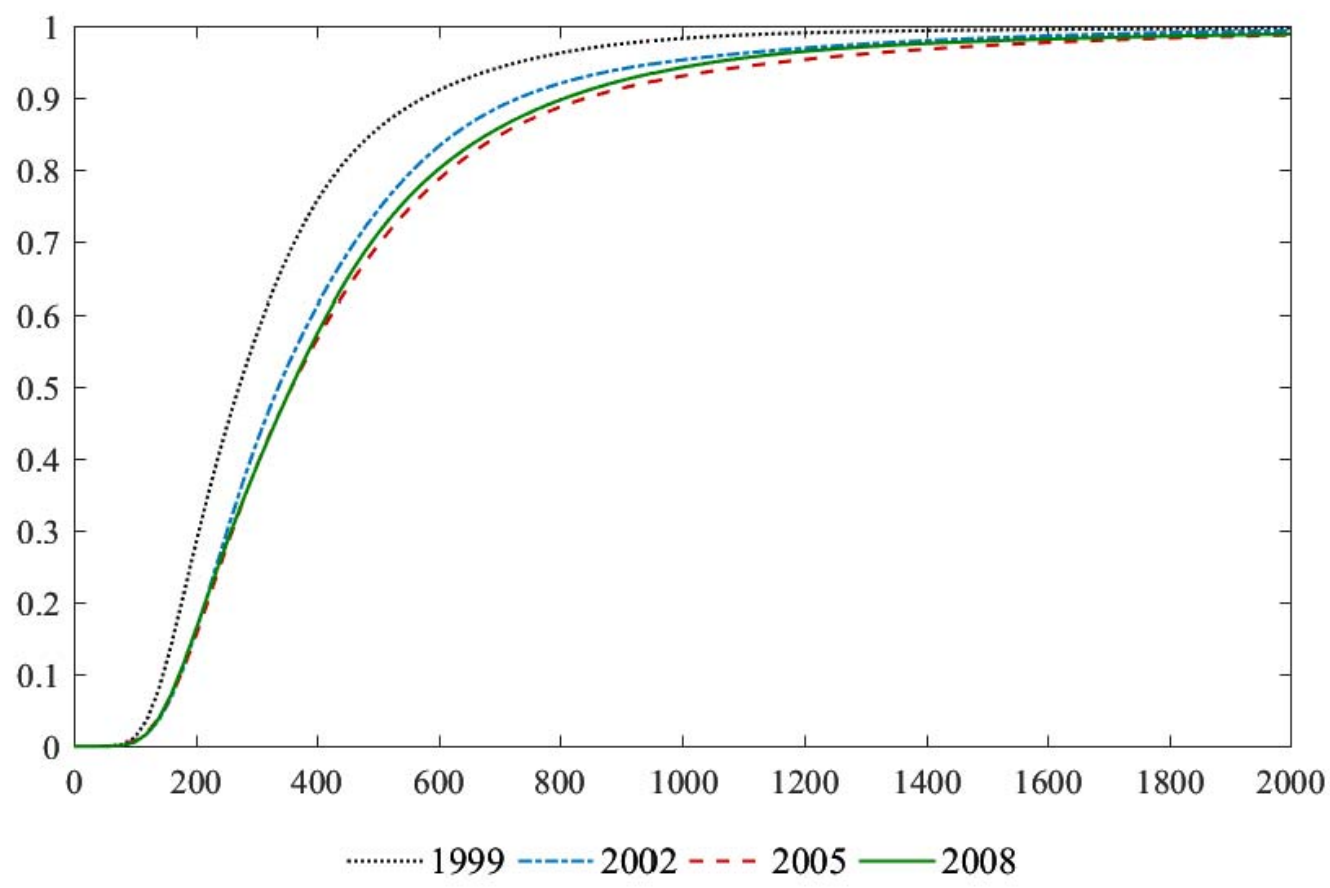

Figure 6: Estimated generalized Lorenz curves with parameters set at posterior means

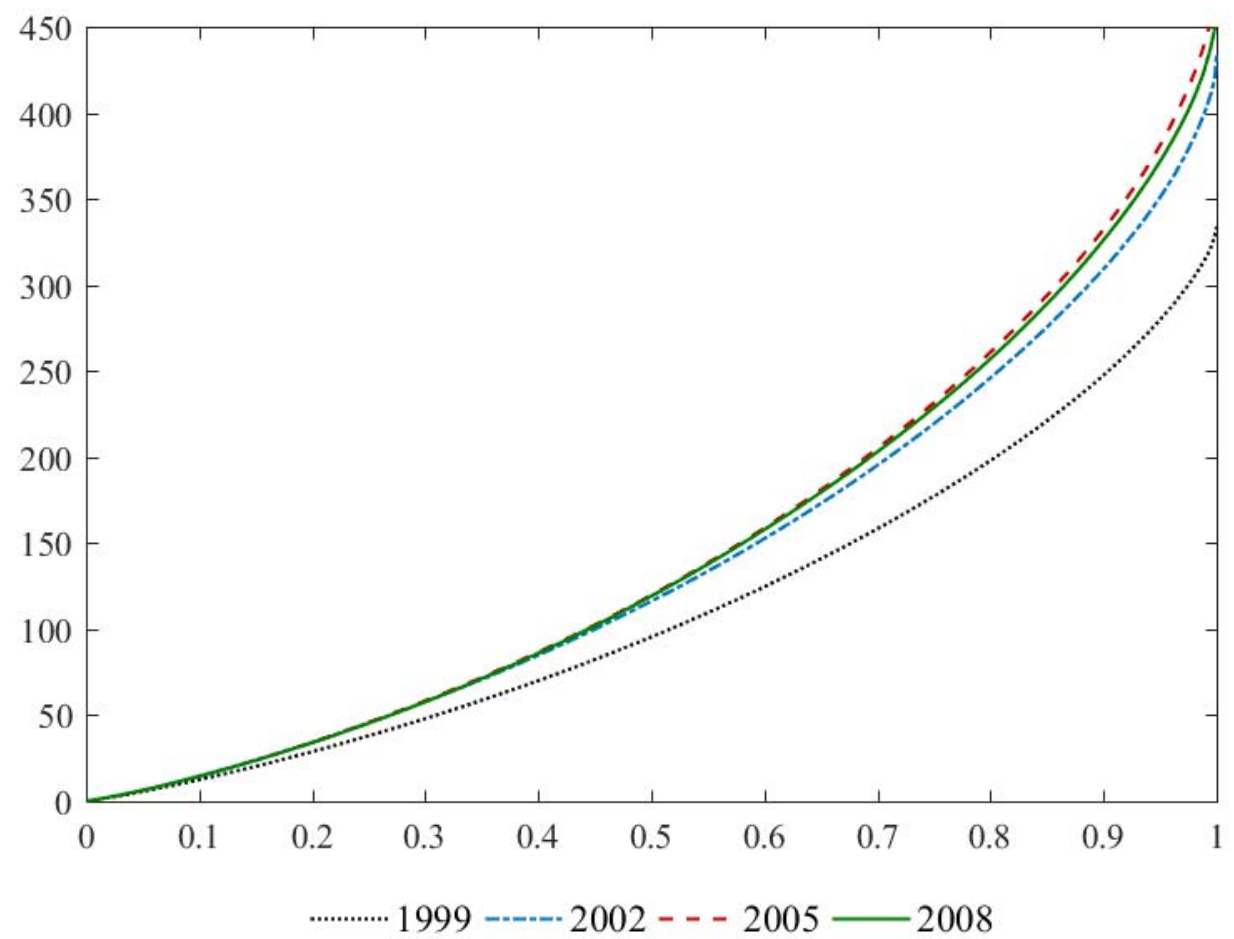


Figure 7: Estimated Lorenz curves with parameters set at posterior means

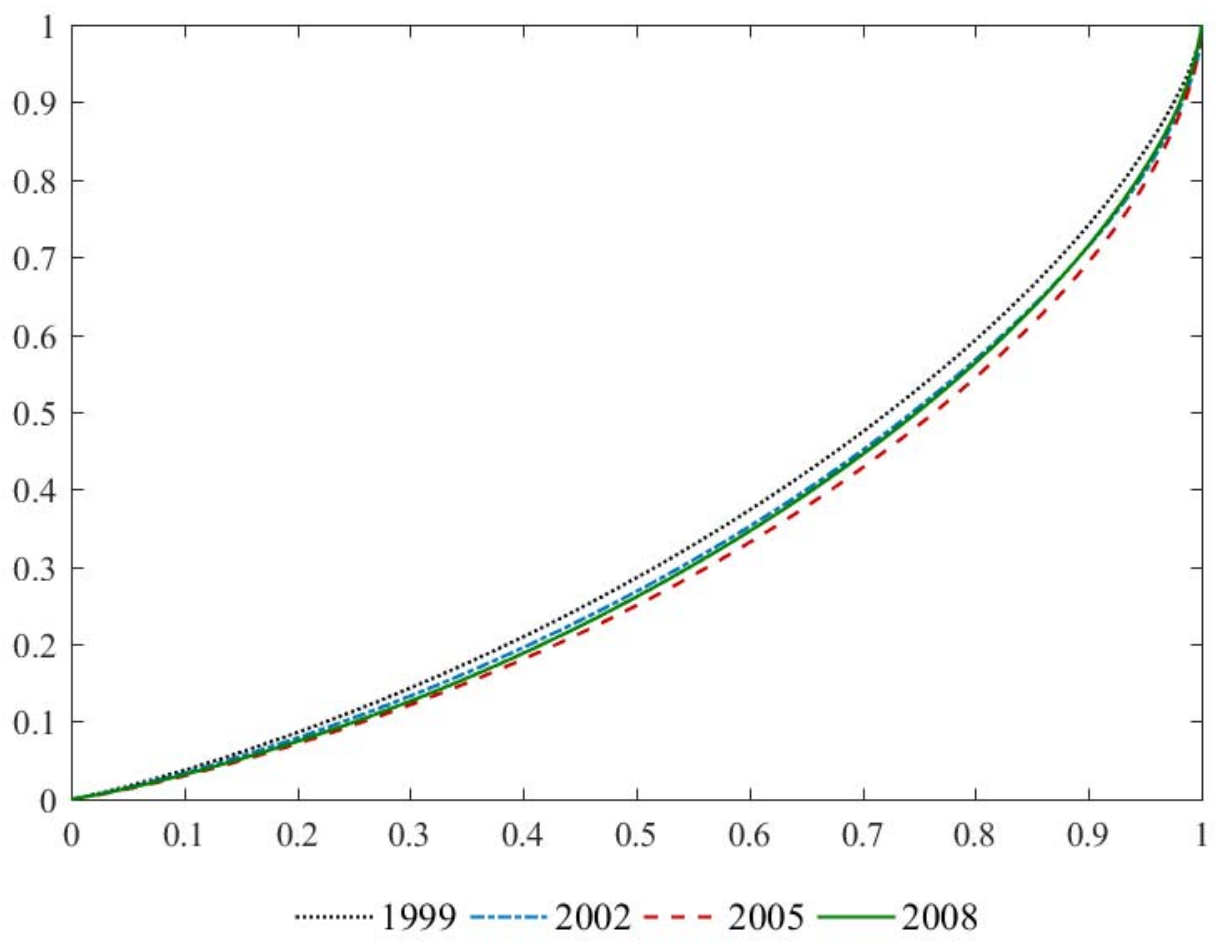


Figure 8: Estimated probability curve of FSD, GLD, and LD for the comparison of 1999 and 2005, with their lower and upper bound
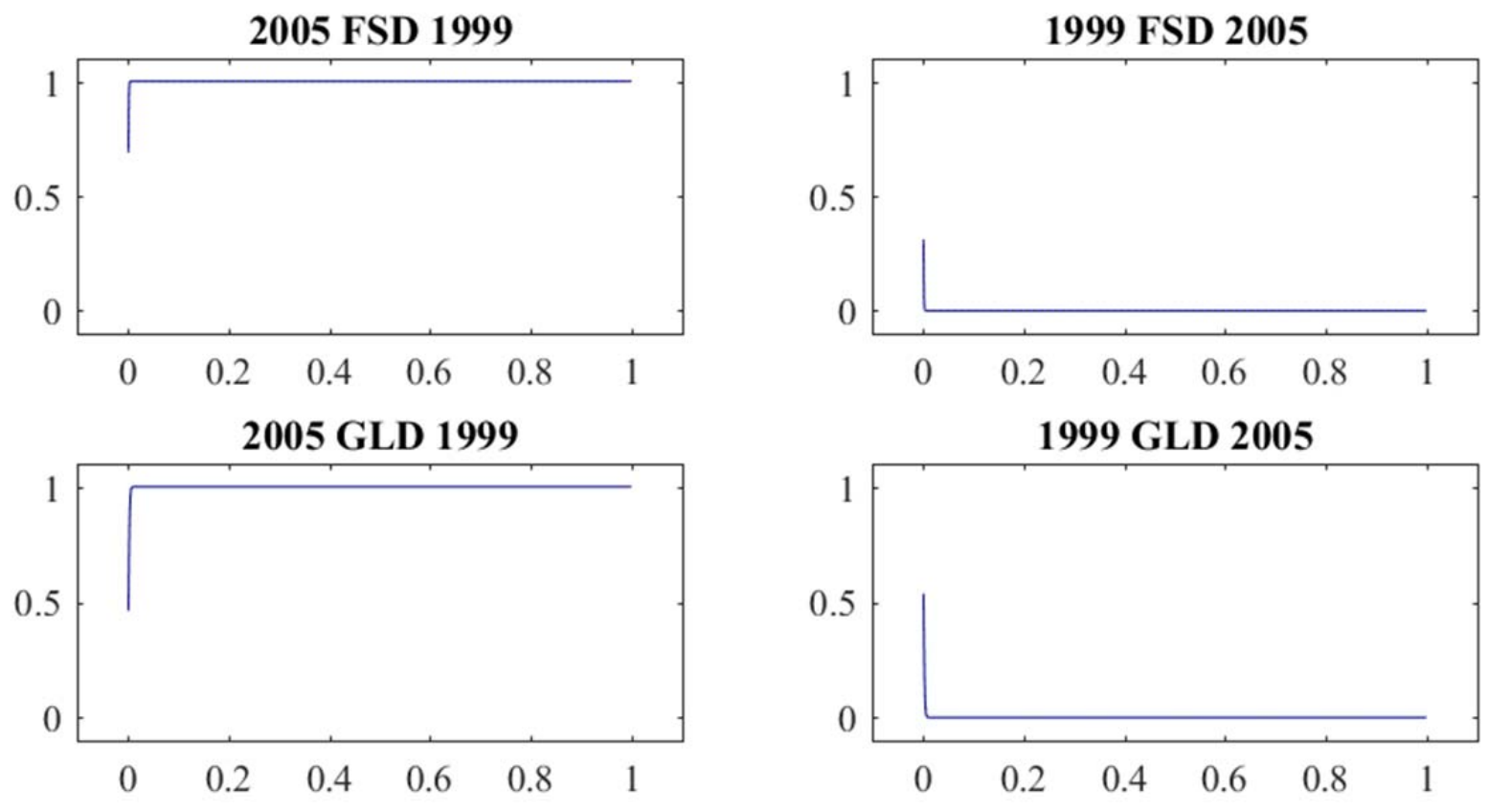

2005 LD 1999
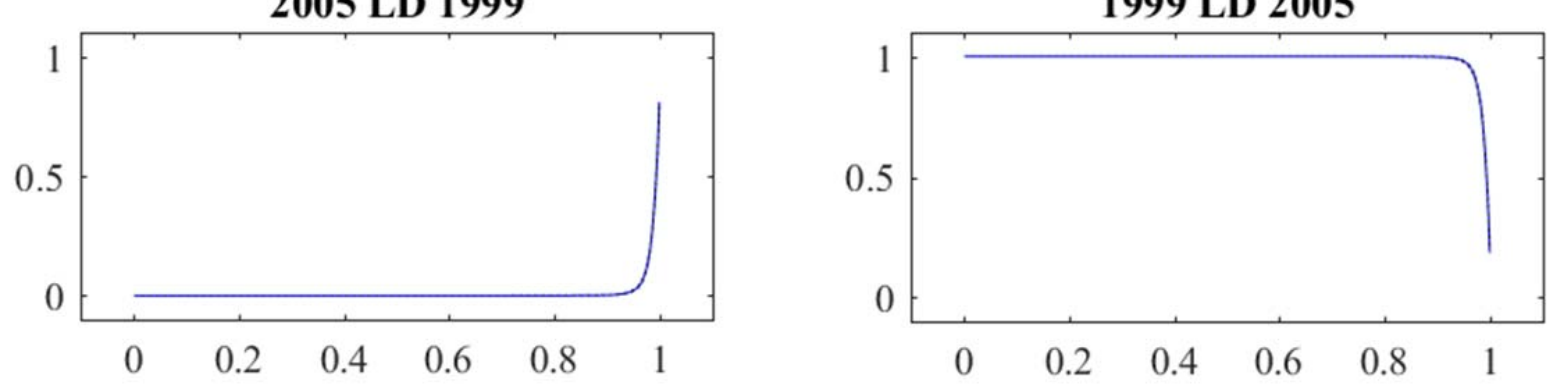

— probability …….... bounds 
Figure 9: Estimated probability curve of FSD, GLD, and LD for the comparison of 2002 and 2005, with their lower and upper bound

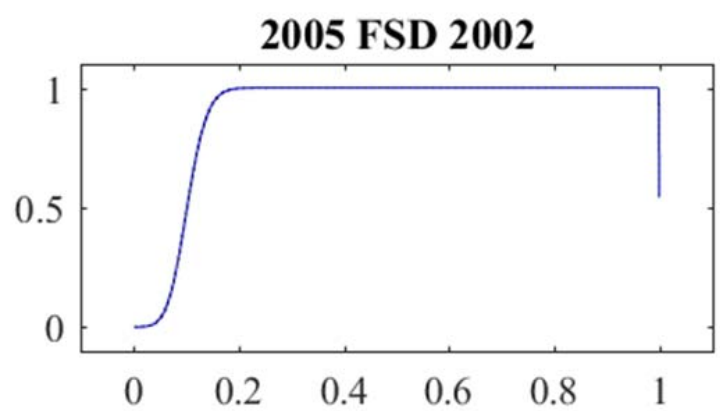

2005 GLD 2002

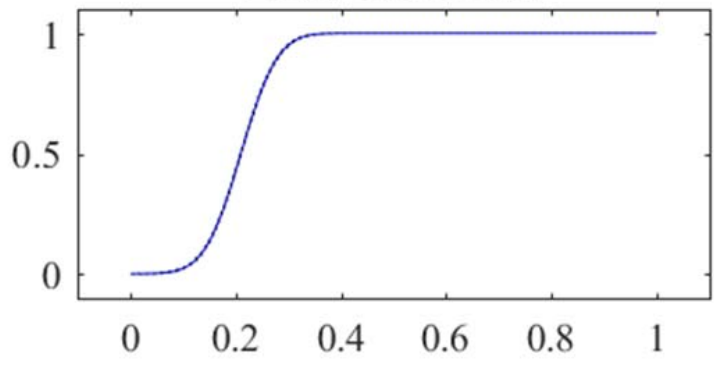

2005 LD 2002

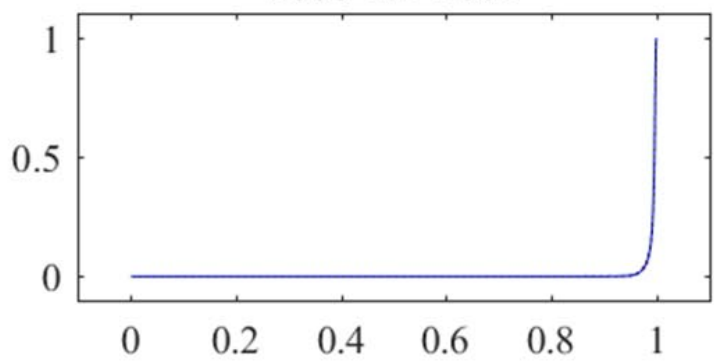

2002 FSD 2005

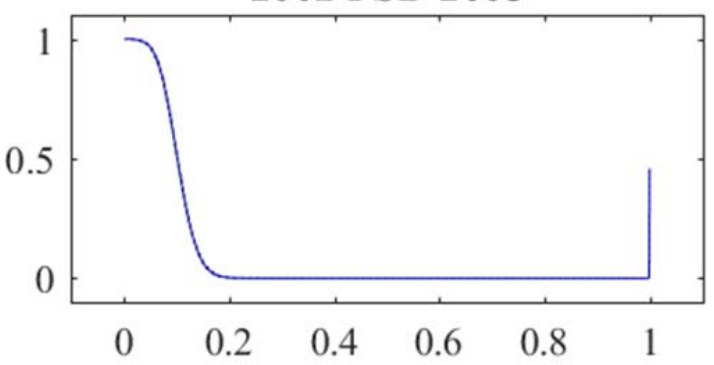

2002 GLD 2005

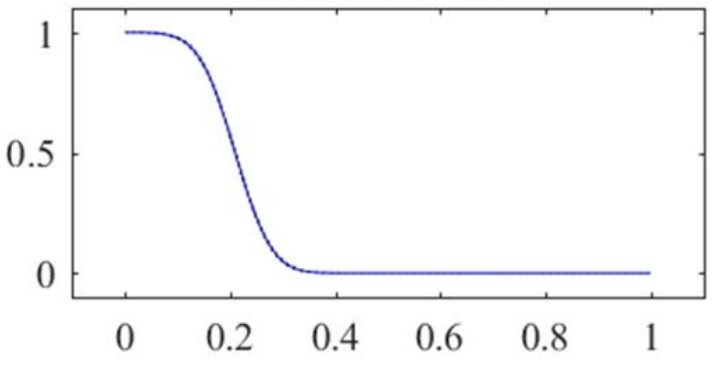

2002 LD 2005

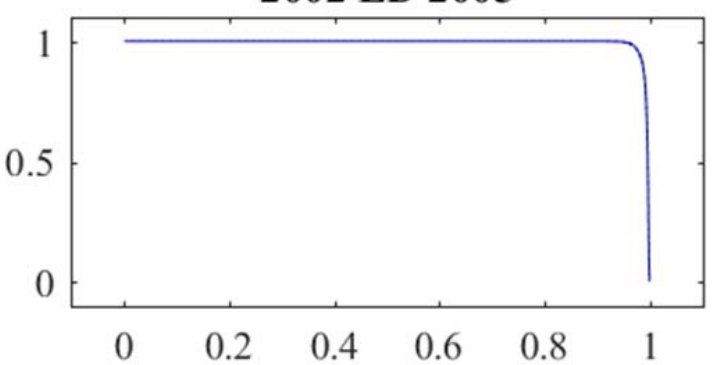

bounds 
Figure 10: Estimated probability curve of FSD, GLD, and LD for the comparison of 2005 and 2008, with their lower and upper bound
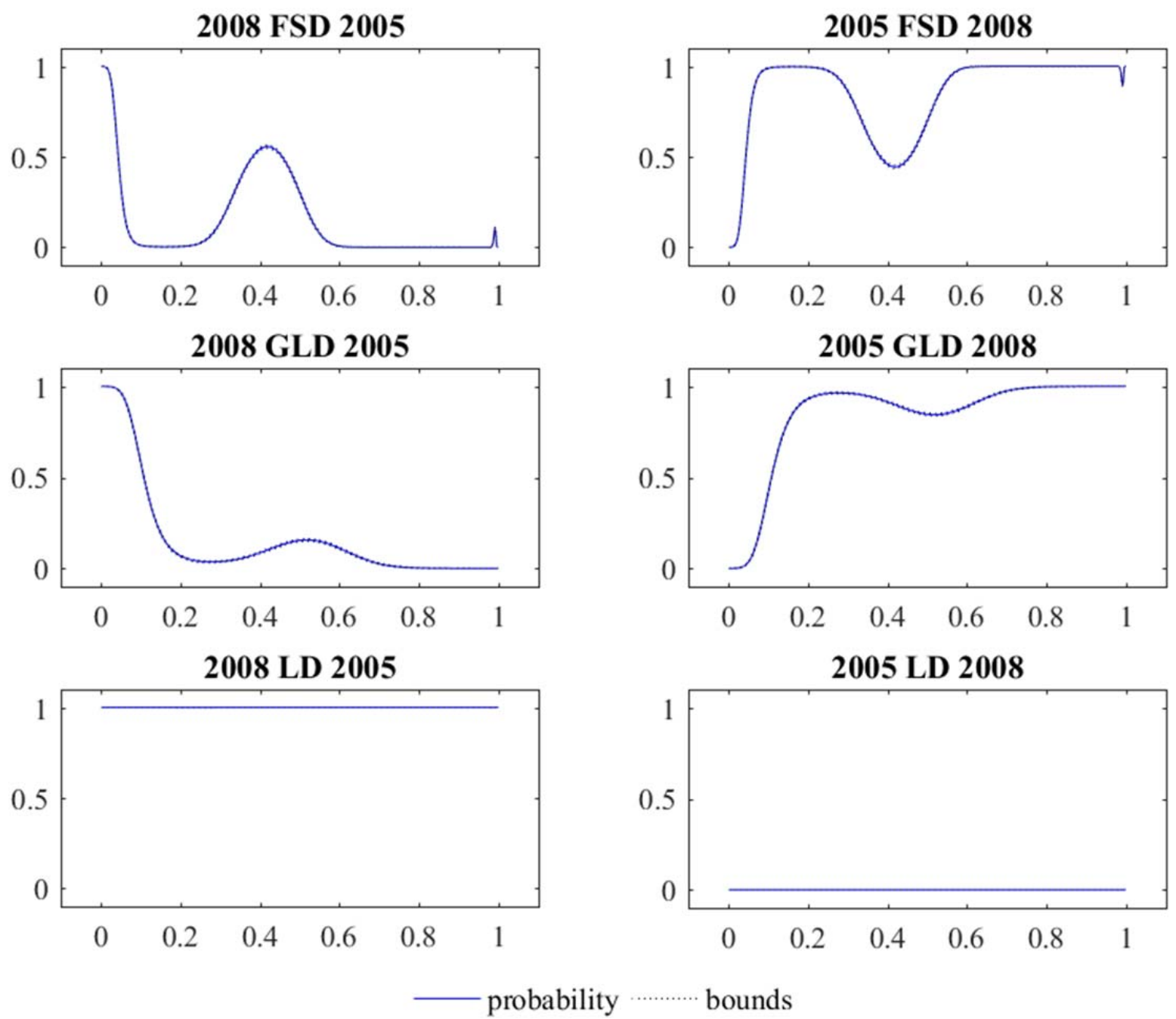